Carleton University

\title{
Psychotherapeutic Drug Use and Technologies of the Self: A study of the intersection of bio-power and nihilism
}

\author{
A thesis submitted to the \\ Faculty of Graduate Studies and Research \\ in partial fulfillment of \\ the requirements for the degree of \\ Master of Arts
}

Institute of Political Economy

by

Todd Culbert

Ottawa, Ontario

January 2005

(C) Todd Culbert 


$\begin{array}{ll}\begin{array}{l}\text { Library and } \\ \text { Archives Canada }\end{array} & \begin{array}{l}\text { Bibliothèque et } \\ \text { Archives Canada }\end{array} \\ \begin{array}{l}\text { Published Heritage } \\ \text { Branch }\end{array} & \begin{array}{l}\text { Direction du } \\ \text { Patrimoine de l'édition }\end{array} \\ \begin{array}{l}\text { 395 Wellington Street } \\ \text { Ottawa ON K1A 0N4 }\end{array} & \begin{array}{l}\text { 395, rue Wellington } \\ \text { Ottana ON K1A ON4 } \\ \text { Canada Oa }\end{array}\end{array}$

Your file Votre référence ISBN: 0-494-00717-6

Our file Notre référence

ISBN: 0-494-00717-6

NOTICE:

The author has granted a nonexclusive license allowing Library and Archives Canada to reproduce, publish, archive, preserve, conserve, communicate to the public by telecommunication or on the Internet, loan, distribute and sell theses worldwide, for commercial or noncommercial purposes, in microform, paper, electronic and/or any other formats.

The author retains copyright ownership and moral rights in this thesis. Neither the thesis nor substantial extracts from it may be printed or otherwise reproduced without the author's permission.
AVIS:

L'auteur a accordé une licence non exclusive permettant à la Bibliothèque et Archives Canada de reproduire, publier, archiver, sauvegarder, conserver, transmettre au public par télécommunication ou par l'Internet, prêter, distribuer et vendre des thèses partout dans le monde, à des fins commerciales ou autres, sur support microforme, papier, électronique et/ou autres formats.

L'auteur conserve la propriété du droit d'auteur et des droits moraux qui protège cette thèse. $\mathrm{Ni}$ la thèse ni des extraits substantiels de celle-ci ne doivent être imprimés ou autrement reproduits sans son autorisation.
In compliance with the Canadian

Privacy Act some supporting forms may have been removed from this thesis.

While these forms may be included in the document page count, their removal does not represent any loss of content from the thesis.
Conformément à la loi canadienne sur la protection de la vie privée, quelques formulaires secondaires ont été enlevés de cette thèse.

Bien que ces formulaires aient inclus dans la pagination, il n'y aura aucun contenu manquant. 


\begin{abstract}
We have invited nihilism into our house. Health, being a designation of an individual's coping strategies to inner anxieties and external stressors, has been monopolized by the health care industry, in turn decreasing individual health. The proliferation of mood and personality-altering drugs within the last forty years is an expression of both the latent consequences of consumer society as well as the medical classification of aspects of the human condition itself. More specifically, mood is the sight at which modern medicine intervenes in the lives of individuals; mood has become a gauge for social integration. Psychotherapeutic drugs are a modern technology of the self whereby individuals can "cosmetically" alter their personalities in socially mandated ways.
\end{abstract}


"Oh wonder, how many goodly creatures are there here, how beauteous mankind is, oh Brave New World that has such people in it."

William Shakespeare

The Tempest

"In sooth, I know not why I am so sad. It wearies me, you say it wearies you, but how I caught it, found it, or came by it, what stuff 'tis made of, whereof it is born, I am to learn; and such a want-wit sadness makes of me that I have much ado to know myself."

William Shakespeare

The Merchant of Venice

"What's the use of their having names," the Gnat said, "if they won't answer to them?"

"No use to them," said Alice, "but it's useful to the people that name them, I suppose."

Lewis Carroll

Through the Looking Glass

To know in the scientific sense means, ultimately, to-have-power-over. To the degree that human beings are authentic persons, unique and self-creating, they cannot be scientifically known.

W.H Auden

Who are we? Where do we come from? Where are we going? What are we waiting for? What awaits us?

Ernst Bloch

The Principle of Hope

Nihilism stands at the door: whence comes this uncanniest of all guests?

Friedrich Nietzsche

The Will to Power 


\section{Table of Contents}

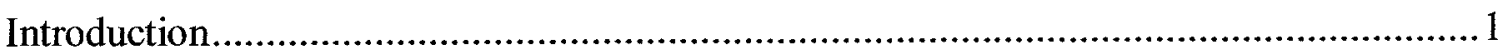

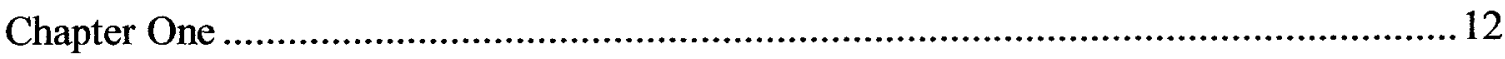

The Global Burden of Disease Project ..................................................................... 12

Approaches to Mental Illness.................................................................................18

The Diagnostic and Statistic Manual of Mental Disorders (DSM) ...............................22

Making Up People ............................................................................................ 41

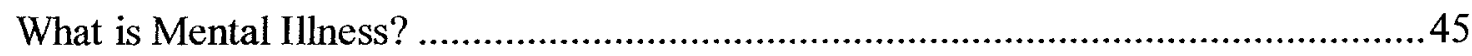

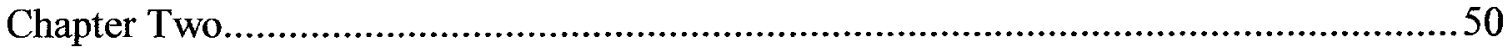

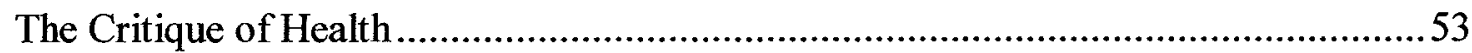

National Work-Life Conflict Study......................................................................... 70

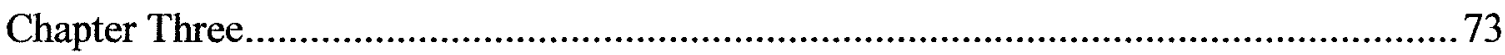

The Pharmaceutical Industry ..................................................................................73

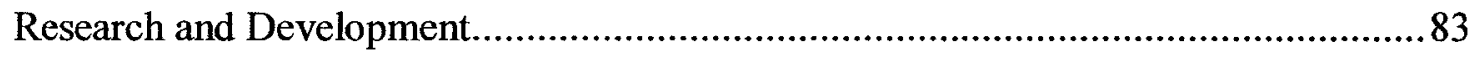

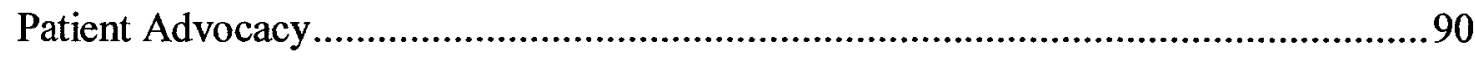

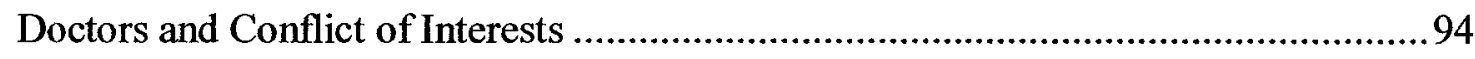

Chapter Four

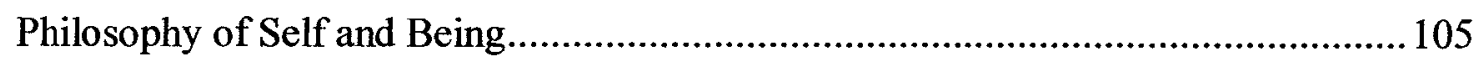

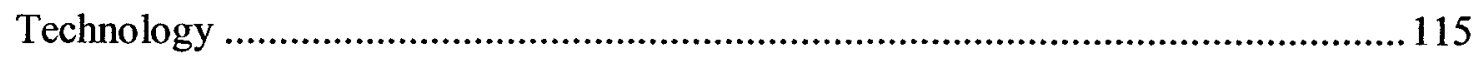

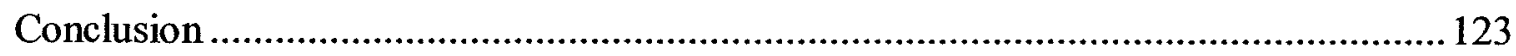

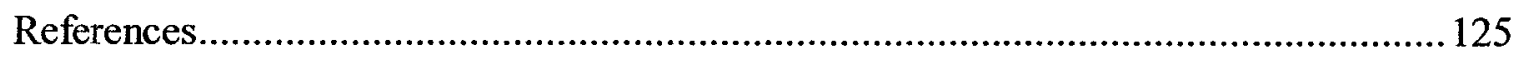




\section{Introduction}

In Canada, a prescription for a mood-altering drug is filled every second of every day (2003 IMS Health Report). In 2002, Canadians spent over a billion dollars on moodaltering psychotherapeutic drugs and roughly one million Canadians are estimated to be clinically depressed (2003 IMS Health Report). In the US, the statistics are only more unsettling. One-fifth of the US population is thought to be suffering from some sort of mental disorder, and at least half of the entire US population will experience some form of mental disorder within their lifetime (Horwitz 2002: 3).

How has modern society, with its apparent wealth and luxury, its advanced means of commodity production and high level of consumerism, become so dependent on drugs that attempt to quell loneliness, anxiety and despair? This thesis will try to understand how the proliferation of psychotherapeutic drug use has altered conceptions of what it means to be a self in modern society. Ultimately, this is a thesis trying to uncover the interaction between powerful mood altering, personality-changing drugs that have been developed within the last 40 years by an industry dominated by the concern for global profits and the social order concerned with an ordering and categorization of individuals through determinants of health.

Beginning my research, I was curious to know if various technologies were emerging within society that were altering the personalities of individuals. By the time I had completed my research, it was obvious the answer was indeed yes. I also wondered if the social discontent that appeared to be evident within modern life was a product of a society in decline, a society plagued with narcissism, alienation and nihilism, or if this 
apparent social discontent reflected in the dramatic numbers of people taking psychotherapeutic drugs was rather the result of an emerging way of looking at society and its mental health, one that increasingly encompassed more and more of the human condition, making rather universal aspects of human existence such as loneliness and anxiety, objects of knowledge and medicalizing them as "unhealthy", thus preventing individuals from pursuing autonomous solutions to the unique life events giving rise to these emotions. The human condition can be defined as those social and cultural determinants that allow for individual human personality development. Humans are born into prearranged structures of meaning and possibilities for action. The human condition is a term for all of these variables taken together as they present themselves to human individuals in their movement through life. For a time I believed these questions to be antithetical. Either society was in decline and the emotional lives of most North Americans were a reflection of hopelessness and despair, or alternatively, quite normal aspects of the human condition were being medicalized through newly emerging technologies of the self in the form of mood and personality altering drugs, leading to the appearance of discontent in the populace at large. However, after some thought, I came to see that these realities are both present. This fact, as expressed through the proliferation of psychotherapeutic drugs, I believe to be an intersection of nihilism and technologies of the self.

The story I want to tell encompasses two plots - nihilism and technologies of the self - converging and interacting, expressing themselves through individuals and the perpetuation of ill mental health throughout society. 
According to Foucault, bio-politics is "that endeavor, begun in the $18^{\text {th }}$ century, to rationalize the problems presented to governmental practice by the phenomenon characteristic of a group of living human beings constituted as a population: health, sanitization, birthrate, longevity, race..." (Foucault 2002: 73). In this way, medical practice becomes concerned with normalization and optimization. Of course, this control need not necessarily be repressive and can lead to positive expression of human action. However, in a society grounded in innately nihilistic conceptions of human value, biopower comes to mobilize the incongruity between the health of individuals and the ill health of society expressing itself through individuals. At the same time as it "creates" health it perpetuates pathology. Each becomes an aspect of the other. While it is true that the medical community does to a large extent create artificial categories of mental illness, it is also true that this is a result of bio-power mobilizing general disenchantment throughout society due to nihilistic ideas of human value (of which medicine its itself a part) in order to reconstitute subjects within society so as to make them understandable; governable. Mental illness - whether "real" or imagined - becomes a technology to be mobilized in the normalization of subjects.

Within the last forty years, bio-power (as a medical power over life and its bodily processes that can be used for political purposes, though this political aspect is not explored in detail within this thesis) has intervened in the lives of individuals through the creation of a technology of the self in the form of magic bullet pills that resocialize human individuals in "socially appealing ways". Psychotherapeutic drugs are prescribed to make individuals more active, more personable, more out-going and optimistic. It is my thesis that mood and personality are being significantly altered through the medical 
technology of self-transformation induced by psychotherapeutic drugs in an attempt to reconcile individual emotional life and the social reality individuals are faced with on a day-to-day basis. I make the assumption that mood (or in general, emotion) is an expression of an individual's relationship to the world; a way of gauging one's "being-in the-world." However, with the rise in the use of prescribed medications that alter mood and personality, mood itself has become a site within individuals to be manipulated and influenced by the medical community to "readjust" individuals within the world. Mood and personality are the sites upon which the technology of the self created by psychotherapeutic drugs alters individual senses of self. The consequences of this are that mood and personality, as expressions of how individuals cope and are socialized over the course of their life, have been cut off from the world upon which they depend and have become gauges for social integration.

Psychotherapeutic drug use complements a society without significant ground upon which individuals can express their humanity apart from the dictates of capital accumulation. To be human is to project value and meaning within the world. In modern society, centered upon the creation and manipulation of desires through marketing and advertising and the mass consumption it entails, life itself has come to be measured, evaluated and judged according to economic principles of value. Individuals lead their lives by the dictates of accounting principles, calculating the costs and benefits of their public and private actions. Life itself has become a cost/benefit analysis: such is the modern human condition. However, this highest value of our society comes to devalue itself in the same way that all values (as points of view toward which action is based outside human control) necessarily end up devaluing themselves, leading to a nihilistic 
conception of the innate valuelessness of life itself. This is the ground upon which postmodern relativism and skepticism have become so dominant. As with all values which posit meaning outside the realm of individual human control, medical values, in their creation and pursuit of health as something given to individuals through the medical community and its technologies, come to devalue themselves as ill health. Or as Ivan Illich wrote (1976), the medical community becomes a threat to health itself.

These two vectors - consumer society with its creation of anxiety and desire, and the health community's pathologising of individual coping mechanisms within such a system - meet and intersect in market society and flesh themselves out through individual emotional reactions within it. The result is "real" mental illness. The next two chapters are a discussion of these two matrices.

And so if it is stated that one in four adults may experience some form of mental illness in their lifetime, what are we implying about modern society, about the individuals who compose it? If it is claimed that three million people within Canada alone suffer from what is termed "severe depression", and that fifty million Americans develop some sort of mental disorder a year (about one fifth of the total population) (CBC Documentary: The Passionate Eye, December 6 2003), what is to be done with such information? Indeed, what has been done with it? These statistics and the corresponding grouping of individuals according to their perceived "level" of health are the driving impulse of this thesis. We now live within a society, indeed an entire world according to World Health Organization data, consumed by mood and personality altering medications, quick fixes to ailments thought to be as old as time, such as depression, and to afflictions originating only within the last 40 years, such as high blood pressure and 
panic disorder, and disorders that appear to have arisen only yesterday, such as erectile dysfunction and generalized anxiety disorder. There has been a creation of discrete disease entities for a plethora of afflictions the likes of which no society has ever known. The creation of disease and illness in our society is so pervasive that it transcends the social and enters the realm of the cultural: we live within a "culture of morbidity" as Ivan Illich puts it. And this creation of disease has been so successful that the pharmaceutical industry, largely insignificant before the Second World War, is now the second most profitable industry in the world, behind only the oil industry.

I came across these problems by initially asking myself what "generalized anxiety disorder" meant, where it came from and where is it taking present conceptions of the self. Generalized Anxiety Disorder is claimed to be a new "disorder", somehow related to, yet also somehow conceptually different from all the others having recently emerged in the psychiatric community and popular consciousness: Major Depressive Disorder; Social Anxiety Disorder; Seasonal Affective Disorder; Panic Disorder; Acute Stress Disorder; Post-Traumatic Stress Disorder. Further, I wanted to know why there has been such an uncritical, passive acceptance of these new "disorders" while the drugs produced and marketed to "cure" them are proliferating throughout our society at an unprecedented rate. Of course, I soon discovered that this acceptance of expanding disorders is itself a clue to the questions I was posing. It became obvious that the answers lay fundamentally in two realms: The commodification of health by the medical profession, particularly the pharmaceutical industry, as well as newly emerging ways of viewing and classifying mental disorders as expressed through the American Psychiatric Association's Diagnostic and Statistical Manual of Mental Disorders (DSM). Here, I found that the penumbra of 
"real" medical conditions has grown from 112 mental disorders in 1952, the first year of the DSM's publication, to 374 by 1994 , the year of its $4^{\text {th }}$ edition. The DSM has been the single largest contributor to the perpetuation of mental illness in its attempt scientifically to classify all possible symptoms attributable to mental disorders, relying almost completely on biological models of mental illness. As I will show, this attempt has been less than successful and has had the repercussion of categorizing aspects of the human condition itself as forms of mental illness.

It was with these questions that I began to sense something ominous with our emerging brave new world. While directly implicating psychiatric practice and the pharmaceutical industry, at the same time I began to think what all this implied for human existence. In light of the proliferation of psychotherapeutic drugs, which often renew an individual's mood and personality in socially appealing ways (Kramer 1997), I wondered what this meant for human beings generally. This led me to the discipline of Ontology and onto the work of Martin Heidegger and Michel Foucault.

In 1927, Heidegger opened his treatise on fundamental ontology by stating that the question of Being has been forgotten. While the difficulties of Heidegger's language may be annoying, even frustrating, Heidegger's lifelong project is fairly straight forward. All Heidegger is trying to claim is that our modern understanding of human beings has become too caught up in theoretical constructions, so much so that philosophers no longer ask the fundamental question of all philosophy: What does it mean to exist at all, when it is perfectly reasonable to assume nothing could very well exist (or in this case not exist) instead? This question has been buried and forgotten through the ages by what Heidegger will call an "ontic" understanding of Being, whereby the self is rendered as an 
object and not a part of the world which is its presupposition. His alternative is an ontological understanding, which takes as its starting point a consideration of the question of what it means to exist at all. Without cascading into the depths of Heideggerian philosophy, into Heidegger's at times impenetrable language and mystic pretensions, I simply want to show in this thesis that modern medicine itself is a product of the very thing that has made modern existence nihilistic, or without foundational meaning, in the first place: the scientific categorization of life itself, including the human subject, which is the direct consequence of an ontic understanding of the world.

This model is in line with Ivan Illich's thesis (1976) that modern medicine has become a threat to health itself, not only through its various practices and techniques, but also through its means of categorizing the human subject and its bodily illnesses. What Illich calls "iatrogenesis", or doctor induced harm, is reflected through modern psychiatric practice.

Because of the domination of modern nihilism, of this ontic understanding of ourselves, we are no longer able to access any ontological understanding of ourselves we have forgotten what it "means to Be". We are classified into categories and subcategories depending on the ways in which we deal emotionally with our human condition. We are "depressed", have "anxiety disorders", "social phobias", are "schizophrenic" etc. And these are thought of as "diseases", as if composed of microorganisms that have infected us from the external environment around us, as if these emotions are biological constructs which have nothing to do with the society within which we live. Mental illness is currently seen by the majority of the medical profession as a natural outcome of biology, of brain dysfunctions, and is treated as such; not as a 
product of social (dis)order. This current view of mental illness could be awkwardly termed the "biologicalization of social effects upon the self". I am of the view that a society dominated by bio-power can never be healthy enough; indeed, it must continually create new forms of illness, disease and disorder in its unending pursuit of health and normalization. Illness creation is implicit in the drive for complete and total health.

Heidegger's analysis of what it means to $\mathrm{Be}$, and how to confront this issue is the impetus for the direction of Foucault's work, as well as for that of a significant portion of the work done by the Frankfurt school. The link between Enlightenment rationality (which is today reflected most radically in the medical profession) and Foucault's technologies of the self seems to lie with Heidegger, so it is with him that I spend some time sorting these issues out. Where Heidegger is asking about the existential condition that he terms "Dasein", or culturally bound, self-reflective and purposive human existence, Foucault is asking about the creation of subjectivities. For my analysis, I see their work as complementing each other. Indeed, I see Foucault's work arising out of the analysis of Heidegger. Through the use of Heidegger and Foucault, it is apparent that selfhood is being reconstituted by the medical community, the implications being a furthering of a nihilistic understanding of what it means to be human, leading to, and exaggerating, a narcissistic concern with mood over any adequate personal understanding by individuals themselves. The personal meaning of life itself is being appropriated by the medical community and is increasingly becoming subject to technologies of the self claiming that aspects of the human condition are disorders in need of intervention and correction. 
Chapter one presents a discussion of the American Psychiatric Association's Diagnostic and Statistical Manual of Mental Disorders - the bible of biological psychiatry and the dominant theory governing current psychiatric practice. The argument pursued here is that only with the reconfiguration of psychiatric practice away from a dynamic model, which views mental disorders as expressions of internal conflicts and is concerned not with the treatment of symptoms but greater self-understanding, towards a symptom-based model assuming that mental disorders are the results of brain malfunctions, allowed for the proliferation in the use of psychotherapeutic drugs.

Chapter two attempts to understand individual and social discontent within consumer society. The argument here is that we live within a nihilistic culture of narcissism, whereby the creation and perpetuation of desire has led individuals increasingly to lose touch with their emotion - the very thing that gauges individual life, thus leading to "actual" ill mental health. The idea of "cultural narcissism" is employed as a means for understanding how emotion and mood connect individuals to the external environment and social world of which they are a part. Narcissism, as the dominant personality trait in consumer culture, is a model implying that the individual self and the external world have merged so completely that individuals can no longer understand themselves within it. The vantage point of objectivity is diminished and emotion becomes something uncontrollable, thus giving rise to such things as panic attacks and anxiety disorders. I have set myself a difficult task in this thesis: trying to find the social reasons for metal disorders at the same time as uncovering the fact that to a large extent mental disorders are the creations of the pharmaceutical and psychiatric medical communities. Chapters one and two, while appearing to be in contradiction, attempt to 
understand, in their own ways, how each vector of social reality has contributed to the proliferation of psychotherapeutic drugs throughout society.

Chapter three undertakes an analysis of the pharmaceutical industry as it pertains to the proliferation of psychotherapeutic drug use. The argument here is that through the control of clinical trials, as well as the marketing of copy-cat drugs to increase market share, have had the direct consequences of contributing to the creation of illness and its "treatment" through chemical intervention. In the drive to expand market share, the pharmaceutical industry has expanded the realm of symptoms that can be attributable to mental disorders in quite the same ways as the American Psychiatric Association.

Chapter four concludes these arguments by discussing the consequences of these occurrences for individual conceptions of self. While presumably "sense of self" is an innately individual terminology, the argument pursued here is that mood, as an optic needed for any sense of self, as well as self-understanding is being normalized and controlled through the use of psychotherapeutic drugs. Psychotherapeutic drugs are a technology of the self, a form of bio-power, allowing individuals to recreate themselves in accordance with their individual reality. However, this is done at the expense of knowledge and understanding of self. Psychotherapeutic drug use, as an expression of the needs of individuals to resocialize themselves in difficult circumstances, only further contributes to the nihilistic conception of modern life from which it came. 


\section{Chapter One}

\section{The Global Burden of Disease Project}

There are such things as mental disorders. Yet, it is also true that to a large extent the number of people claimed to have mental disorders depends upon ways of classifying and the means of measuring mental disorder. This chapter will attempt to work out the problems associated with the psychiatric community's classification and standardization of mental disorder. The argument pursued is that in the quest for diagnostic reliability biologically-based psychiatry ignored claims of validity and ended up expanding the realm of what actually constituted mental disorder. Further, with the assumption that there is little causal relation between mental disorders and an individual's life history, that disorders are simply products of chemical dysfunctions in the brain, this model allowed for and legitimated mass prescribing of psychotherapeutic drugs. Chapter two will attempt to understand the social and cultural forces leading to "real" mental disorder.

Social life is dominated by classification and standardization; indeed, classification and standardization are integral to social life. To classify, to create models out of social phenomena, allows individuals and societies to better negotiate social reality. From library catalogue systems to the sorting of whites from darks before we wash our clothes, classificatory systems consume individual and social life. However, as Geoffrey Bowker and Susan Leigh Star write in Sorting Things Out: Classification and its Consequences (1991), often these standards and classifications are invisible precisely because they are so ubiquitous in social life. Invisibility results when classification and standardization become so hegemonic we no longer recognize that we are even classifying and standardizing at all. And while this invisibility is trivial when it comes to 
hot or cold wash cycles, it has much more profound consequences when classifications and standardizations are applied to individuals. This chapter will attempt to uncover the classificatory invisibility within the psychiatric community, how it has come about and what its implications are. The thesis pursued here is that with the rise of diagnostic, symptom-based psychiatry, chemical intervention in the form of psychotherapeutic drugs became a legitimate medical and psychiatric practice, allowing for the perpetuation and increase in the use of these drugs throughout society. Yet the full social consequences (which are often negative, increasing the likelihood of chemical dependence, suicide, violence and aggression in patients) of the rising numbers of individuals on psychotherapeutic drugs are only now beginning to be fully understood.

According to Bowker and Star, classification and standardization are, in an ideal sense, composed of four aspects: ubiquity; materiality; historical indeterminateness; and politics. The first two aspects of classifications and standardization seem fairly selfevident: classification and standardization are everywhere one chooses to look, indeed are themselves a very part of "looking" at the world. However, the last two are relevant to my discussion on modern psychiatric practice. By historical indeterminateness, Bowker and Star mean that through classificatory systems we are continually in the process of writing and rewriting knowledge of the past in light of new developments in the present. As well, through the aspect of the politics involved in the creation and refinement of systems of classification, the authors highlight that the battle for classification creation and its criteria is by no means easily accomplished, often only achieved through negotiation and/or force. Both historical indeterminacy and political negotiation are 
involved in the creation of the DSM, the most well-known manual of mental illness classification.

Alan Desrosieres, drawing on Foucault's work, in his essay "How to Make Things Which Hold Together", discusses how, politically, the collection of statistics on certain population groups itself leads to an idea about that group which "holds it together". Statistics, for Desrosieres, constructs equivalence among diverse objects, creating a visibility of certain populations and of their composition. In the process of statistical data collection, "differences between nomenclatures are problems that must be eliminated" (Desrosieres 1990: 197). For example, Desrosieres cites the creation of crime rates as contributing to the idea of a thing which holds together; namely, the human propensity for murder.

The World Health Organiztion (WHO) has been the single greatest data collection agency for world health. Recently, the WHO has shifted its historical emphasis on physical diseases such as HIV/Aids, malaria, and other fatal diseases to an emphasis on mental health within the world's populations. In the same way Desrosieres posits the use of statistics among diverse objects as creating a totalized idea of similarity, the WHO has, through the use of world statistics on mental health, created something else which holds together: homogeneity of mental illness across different countries and cultures and the disability arising from mental illness. In so doing, the WHO ignores all culturally and socially mediated interpretations of mental disorder as well as differing methods of "treatment"; something many cultures have little need for anyway, since their social structure provides for its inclusion (Diamond: 1977). 
According to the 2001 World Health Report released by the World Health Organization, around 450 million people worldwide suffer from mental, neurological or psychosocial disorders, the most common form of mental disorder being major depression, followed by anxiety disorders, which include panic attacks, phobias and posttraumatic stress disorder (WHO 2001: 6). Further, major depression, according to the WHO classification, is the leading cause of disability globally and ranks fourth in the ten leading causes of what is referred to as the "global burden of disease", a worldwide measure of the effects of physical and mental disease (WHO 2001: 5).

On the world stage, no other organization has been as responsible for the classification and standardization of mental disorders as the World Health Organization. Indeed, this bureaucracy is so large and so well-known that many health care professionals as well as individual citizens within various countries consider its classifications and estimates of world disease to be naturally occurring, and not as the product of classificatory methods produced by the WHO itself. In 1992, The Harvard School of Public Health, in collaboration with the World Bank and the World Health Organization, initiated the Global Burden of Disease project. The study included the first global estimates of disease and injury burden attributable to different risk factors, and involved 100 collaborators in more than 20 countries. Instead of conducting data analysis of the usual variables, such as disease per age group, race, gender, income etc. the project tried to quantify the burden of living with disease. So, instead of attempting to figure out where disease was most prevalent, or how many people were afflicted with different types of disease, the Global Burden of Disease study attempted to quantify the disease 
and injury burden of over 100 conditions and made projections over 30 years for 500 consequences of these conditions.

In the analysis, the WHO tried to make the argument that disease burden involved more than premature death; it also involved living life marred by chronic, debilitating and incapacitating disease. To this end, the Global Burden of Disease project wanted to find a health gap measure that extended the concept of potential years of life lost due to premature death to include years of "healthy" life lost in states of less than full health what the WHO considers "disability".

To this end, the Global Burden of Disease study developed a single measure to allow comparison of the burden of disease across many different disease conditions by including both death and disability. This measure was called Disability Adjusted Life Years (DALYs). DALYs measure lost years of healthy life regardless of whether the years were lost to premature death or disability. The disability component of this measure is weighted for severity of the disability. For example, in 1990 disability caused by major depression was found to be equivalent to blindness or paraplegia, whereas active psychosis seen in schizophrenia produces disability equal to quadriplegia (WHO web site).

Using the DALYs measure, in 1990 major depression ranked second only to ischemic heart disease in magnitude of disease burden in "established" market economies (WHO web site). Schizophrenia, bipolar disorder, obsessive-compulsive disorder, panic disorder, and post-traumatic stress disorder also contributed significantly to the total burden of illness attributable to mental disorders. The projections show that with the aging of the world population and the successful treatment of infectious diseases, 
psychiatric and neurological conditions could increase their share of the total global disease burden by almost half, from 10.5 percent of the total burden to almost 15 percent in 2020 (WHO web site).

So, according to the WHO Burden of Disease project, major depression is the leading cause of disability (measured by the number of years lived with a disabling condition) worldwide among persons age 5 and older. For women throughout the world, as well as those in established market economies, depression is the leading cause of DALYs. In established market economies, schizophrenia and bipolar disorder are also among the top 10 causes of DALYs for women.

One consequence of the WHO disability scale is that it itself creates a perceived world of disability. Mental illness, as something which holds together, is standardized into disability. Whether or not the WHO classifications are "real" or not is secondary in importance to such a vast population believing they are as well as physicians and psychiatrists treating mental illness as if they were. With these kinds of statistics, it would appear that we live in an entire world paralyzed by fear and anxiety, an age of terrorism and insecurity causing many years of lost life due to mental illness. With such an increasingly hegemonic view of mental disorder prevalence and its disabling consequences, the need for "technologies of self" that intervene in the lives of individuals becomes easily justified, both by individuals themselves and society at large. When mental disorder becomes a widespread object of government policy, bio-power's control over individuals increases, leading to a morbid society compulsively focused upon the health and ill-health of individuals. 


\section{Approaches to Mental Illness}

There are three basic models attempting to explain mental health and illness, each differing to the extent to which it emphasizes certain factors over others: the sociological, the psychological and the biological.

Sociological approaches view mental illness as a breakdown of an individual's mental state in the face of overpowering, external stress. Mental illness, in its various forms, is assumed to be attributable to the amount of external stress an individual is faced with throughout their life. These stressors can be effectively heightened by the organization of society itself, as well as by discourses produced and perpetuated throughout society (labeling theory or what Ian Hacking calls "classificatory-looping", discussed below). It is an argument of this thesis that the decreasing popularity of this model was itself necessary for the proliferation of psychotherapeutic drugs.

The second model used to understand mental illness, the psychological approach, focuses attention on the individual factors that produce abnormal thoughts, feelings and behaviors. Until the Diagnostic and Statistical Manual of Mental Disorders became widespread in 1980, Freudian psychotherapy - psychoanalysis - was the most influential paradigm in psychiatry. This model assumed that individuals are closed energy systems motivated by a variety of drives (mainly, the drive of Eros, after the Greek god of love). Because civilization would not be possible if each individual fulfilled the immediacy of their needs, Freud claimed that individuals compromise these drives through the creation of an Ego, which is constantly juggling the desire for immediate gratification (the id, which is responsible for irrational, animalistic tendencies), and the demands of social dictates (internalized in the form of a super-ego). Because of the dominance of these 
drives, present even at birth, repressed and frustrated childhood desires for selfactualization through Eros could lead to neurosis if reactivated in adulthood.

Psychoanalysis, or talk therapy, was a method for releasing this energy, leading to a more "stable" individual.

Finally, biological approaches view mental illnesses as diseases of the brain. With advances in genetics, as well as pharmaceutical advances in available drug therapies, which both contribute to a greater understanding of the chemistry of the brain and neuronal communication, this biological model of mental illness grew in popularity throughout the 1960s and 1970s, finally becoming the dominant paradigm of mental illness in the 1980s. This "revolution" in psychiatry is summed up nicely by Nancy Andreasen, a prominent biological psychiatrist and Editor-in-Chief of the American Journal of Psychiatry, as well as the winner of the National Medal of Science, the highest scientific honor in the US. Andreasen makes it clear in her book entitled Broken Brain: The Revolution in Biological Psychiatry that the shift in perception from individual and social causes to strictly biological causes of mental disorder was not brought about by breakthroughs in knowledge about the etiology of mental disorders, but was based on new conceptions of where to look for etiological factors:

[biological psychiatry] is a revolution not so much in terms of what we know as in how we perceive what we know. This shift in perception suggests that we need not look to theoretical constructs of the "mind" or to influences from the external environment in order to understand how people feel, why they behave as they do, or what becomes disturbed when people develop mental illness. Instead we can look directly to the brain and try to understand both normal behaviour and mental illness in terms of how the brain works and how the brain breaks down. The new mode of perception has created the exciting feeling that we can understand the causes of mental illness in terms of basic biological mechanisms.

(Andreasen 1985: 138) 
It is this new "mode of understanding" I wish to explore further. The goal of biological psychiatry is to define and classify diseases by their particular biological mechanisms, or what is referred to as their "pathophysiology", and to find the causal factors contributing to the mental disorders. However, as Schwartz admits, because the causes of the pathophysiology are in most cases unknown, biological psychiatry relies on diagnosis of mental illness through the use of symptom-based criteria, which appear to form similar patterns in population groups and which help predict the course of illnesses as well as possible treatments for them. This way of looking at mental illness was begun by German psychiatrist Emil Kraepelin in the late nineteenth century. His biological model of mental illness, or what is referred to as "diagnostic" psychiatry, went largely unrecognized with the rise of the Freudian and Neo-Freudian schools of dynamic psychiatry. His framework lay in wait for nearly a hundred years before it was resurrected by a group of psychiatrists revising the DSM. This biological approach suddenly came back to the forefront of the understanding of mental illness in the $1970 \mathrm{~s}$. It did so in the name of scientific accuracy, not through any proof that its method was more successful in "curing" mental disorder. In fact, biologically-based psychiatry increased the number of mental disorders through its symptom-based classificatory mechanisms.

Peter Kramer, psychiatrist and author of Listening to Prozac, the bestselling book that popularized and even condoned the notion of "cosmetic pharmacology", whereby psychotherapeutic drugs are created to alter personality in socially appealing ways, much like plastic surgery but for individual psychology, writes: "In clinical pharmacology, contemporary technology plays a dominant role in shaping ideology. What we look for 
in patients depends to a great degree on the available medications" (Kramer 1997: 35). The creation and subsequent proliferation of specific mental illnesses required a certain type of medical perception to gain hegemony over others, one that needed to be reliable across a large population in ways previous Freudian methods were not. As Bowker and Star write, "the substitution of precision for validity" is often a method implemented for solving ethical debate (Bowker and Star 1991: 24). Further, according to Allan Horwitz, "the reasons for the proliferation of mental illnesses lie in the historical development of the psychiatric profession over the course of the twentieth century and in the useful social functions such an expansive definition performs for a number of different groups" (Horwitz 2003: 37). With the breakdown of the Freudian system (which occurred due to ideological and political causes and not to any advancement in the actual understanding of mental disorders, which I will explain below), biological, "Neo-Kraepelin" psychiatry remerged through claims of being more rigorously scientific than dynamic, Freudian models. This allowed mental disorders to become a problem to be corrected and treated with mood and personality altering medications, rather than previous psychiatric methods, such as talk therapy (whatever one may think of psychoanalysis itself as a psychiatric method of treating mental disorders). However, this did not come before Freudian psychology had opened up the realm of diagnosable psychiatric disorders.

One can see this change through an analysis of the American Psychiatric Association's Diagnostic and Statistical Manual of Mental Disorders (DSM). It is in the DSM that the sheer number of mood disorders grew exponentially. Not only do specific symptom-based classifications increase but so too does the amount of written text trying to explain these disorders in biological terms. Further, with each subsequent revision and 
expansion of the DSM, a great deal of emphasis is placed on how certain disorders are thought to be conceptually distinct from other disorders, even though they may have been at one time thought to be the same. Where did this manual come from and why? To understand more about the reasons for the rise of a new theoretical framework within the mental health community in the late seventies - one that based itself almost solely on traditional, biology-based medical models of symptom classification originating in the work of Emil Kraepelin - one needs to understand the DSM's creation and the social and political factors surrounding, and determining, its revision.

\section{The Diagnostic and Statistic Manual of Mental Disorders (DSM)}

The current reference work in the field of psychiatry, as well as psychiatric epidemiology is the American Psychiatric Association's (APA) Diagnostic and Statistical Manual of Mental Disorders (DSM), first published in 1952, with considerable revisions in $1968,1980,1987$ and 1994 . Its history is of fundamental importance to the understanding of current conceptions of mental illness in the medical community as well as the lay population. With the publication of the DSM III in 1980, a fundamental alteration in how mental illness should be perceived and classified, and correlatively, how it should be treated - particularly through chemical intervention in the form of psychotherapeutic drug use - took place.

Until very recently - beginning no later than the mid 1960's - discrete, conceptually distinct mental illnesses had a very limited role within psychiatric practice (Shorter 1997). This was due, in large part, to the still dominant theoretical model governing how mental illness was perceived: dynamic psychiatry. Dynamic psychiatry rested mainly on the Freudian model of mental disorder. 
The key to the Freudian system is, of course, Freud's creation of a deterministic motivator of human personality and human action called the "unconscious". Freud was perhaps the first psychologist to apply deterministic principles to human mental existence and to hold that human behaviour can only be explained in terms of hidden mental states which determine it (Gay 1999). When Freud published his Interpretation of Dreams at the turn of the nineteenth century, psychiatry was an asylum-based discipline that treated the most serious mental disorders through somatic therapies. Dynamic psychiatry revolutionized the classification of psychiatric symptoms (Horwitz 2003, Shorter 1997) and thus their treatment. Instead of treating the behaviour of mentally ill patients as inexplicable or as the product of brain diseases, Freud claimed that, in treating mentally disordered patients, it was important to seek an explanation by searching for causes in terms of the mental states of the individual concerned. Until the rise of dynamic psychiatry in the late nineteenth century it was "culturally impossible for sufferers from any but the most severe mental disorders to formulate their problems in psychiatric terms" (Horwitz 2002: 40).

Associated with this view of the mind is Freud's account of the instinct, or drives. The instincts, for Freud, are the motivating forces within the unconscious that energize the mind. Freud posited two main instincts motivating human behaviour: Eros, or the life instinct, which gives motivation to the self-preserving and erotic instincts; and Thanatos, the death instinct, which motivates the instincts toward aggression, self-destruction and cruelty (Gay 1999). While to claim that Freud posited all human behaviour in terms of sexual gratification is untrue in light of Thanatos (an element in his system that he did not come up with until late in life and felt much frustration over not having come up with 
earlier), the essence of his theory of instincts is that human beings are energized from birth to acquire and enhance bodily pleasure.

Freud's theory of infantile sexuality is an integral part of his theory of human personality. From his account of the instincts, it followed that from the moment of birth the infant is driven in its actions by the desire for bodily/sexual pleasure. This is seen by Freud as the desire to release mental energy. Initially, infants gain such release, and derive such pleasure, through the act of sucking - the 'oral' stage of human personality development. This is followed by a stage in which the locus of pleasure or energy release is the anus, particularly in the act of defecation, and this is accordingly termed the 'anal' stage. Then the child develops an interest in its sexual organs as a site of pleasure (the 'phallic' stage), and develops a deep sexual attraction for the parent of the opposite sex, and a hatred of the parent of the same sex (the 'Oedipus complex'). This, however, gives rise to socially derived feelings of guilt in the child, which recognizes that it can never displace the stronger parent. In the case of a male, it also puts the child at risk, where he perceives that if he persists in pursuing the sexual attraction for his mother, he may be harmed by the father; specifically, he comes to fear that he may be castrated. This is termed 'castration anxiety'. Both the attraction for the mother and the hatred for the father are usually repressed, and the child usually resolves the conflict of the Oedipus complex by coming to identify with the parent of the same sex. This happens at the age of five, at which point the child enters a 'latency' period, in which sexual motivations become much less pronounced. This lasts until puberty, when mature genital development begins, and the pleasure drive refocuses around the genital area. 
This, Freud believed, is the sequence or progression implicit in normal human development, and it is to be observed that at the infant level the instinctual attempts to satisfy the pleasure drive are frequently checked by parental control and social coercion (Gay 1999: 231). The developmental process, then, for the child is essentially a movement through a series of conflicts, the successful resolution of which is crucial to adult mental health. Freud held that any mental illnesses, particularly hysteria, can be traced back to unresolved conflicts experienced at this stage, or to events which otherwise disrupt the normal pattern of infantile development.

Freud also posited a tripartite model of the mind that tried to account for the mediation of human drives and the social demands that limited them. The id, ego and super-ego where thought to be internal states mediating these basic drives within human beings. If the external world offered no scope for the satisfaction of the id's pleasure drives, or if the satisfaction of some or all of these drives transgressed the moral sanctions laid down by the super-ego, then an inner conflict would occur in the mind between its constituent parts. Failure to resolve these inner conflicts led to neurosis. A key concept introduced here by Freud is that the mind possesses a number of 'defense mechanisms' to attempt to prevent conflicts from becoming too painful. These defense mechanisms included repression (pushing conflicts back into the unconscious); sublimation (channelling sexual drives into the achievement socially acceptable goals, in art, science, poetry, etc.); fixation (the failure to progress beyond one of the developmental stages); and regression (a return to the behaviour characteristic of one of the stages) (Gay 1999: 240). 
Of these, repression is the most important. When a person experiences an instinctual impulse to behave in a manner which the super-ego deems to be reprehensible (ie. a strong erotic impulse on the part of the child towards the parent of the opposite sex), then it is possible for the mind to push it away, to repress it into the unconscious. Repression is thus one of the central defense mechanisms by which the ego seeks to avoid internal conflict and pain, and to reconcile reality with the demands of both id and super-ego. As such, it is completely normal and an integral part of the developmental process through which every child must pass on the way to adulthood.

However, the repressed instinctual drive, as an energy-form, is not and cannot be destroyed when it is repressed - it continues to exist intact in the unconscious, from where it exerts a determining force upon the conscious mind, and can give rise to the dysfunctional behaviour characteristic of neuroses. This is one reason why dreams and slips of the tongue are significant for Freud, and why their analysis became such a key part of his treatment - they represent instances in which the authority of the super-ego is relaxed, and when the repressed drives are accordingly able to present themselves to the conscious mind in a more discernable way. The difference between 'normal' repression and the kind of repression which results in neurotic illness is one of degree, not of kind the compulsive behaviour of the neurotic is itself a behavioral manifestation of an instinctual drive repressed in childhood. Such behavioral symptoms are highly irrational (and may even be perceived as such by the neurotic), but are completely beyond the control of the subject, because they are driven by the now repressed impulse. Freud positioned the key repressions, for both the normal individual and the neurotic, in the first five years of childhood. 
Freud's account of the sexual genesis and nature of neuroses led him to develop a clinical treatment for correcting such disorders: Psychoanalysis. The aim of psychoanalysis is simply to re-establish a harmonious relationship among the three elements that constitute the mind (id, ego and super-ego) by excavating and resolving repressed unconscious conflicts. The task of psychoanalysis as a therapy is to find the repressions that are causing the neurotic symptoms by delving into the unconscious mind of the subject, and by bringing them to the forefront of consciousness, allowing the ego to confront them directly and thus to discharge them.

The end goal of psychoanalysis is to form greater self-understanding. Once this is acquired it is supposed to be up to the patient, in consultation with the analyst, to determine how they should use this newly-acquired understanding of the unconscious forces which motivate them. One possibility is channeling sexual energy into the achievement of social, artistic or scientific goals - sublimation, which Freud saw as the motivating force behind most great cultural achievements. Another is the conscious, rational control of the formerly repressed drives - suppression. Whatever the case, the "cure" to neurosis is thought to be achieved essentially by allowing for a kind of catharsis - a release of the pent-up psychic energy, the constriction of which was the basic cause of the neurotic illness.

The single greatest consequence of the Freudian system of neuroses and human psychopathology for the future classification of mental illnesses was that it fundamentally changed the relationship between mental illness and normality, making the division between the two less sharp (Horwitz 2002). It is this "dynamic" approach to psychiatry, whereby disorders are not discrete disease entities, but simply matters of degree in the act 
of mediating internal drives that allowed for the explanation of many types of abnormal behaviour. In the reduction of human personality to a common source in the unconscious drives of individuals, the realm of possible distinct mental illnesses increased significantly. As David Horwitz writes: "dynamic psychiatry laid the foundation for the sprawling mass of troubling behaviours that diagnostic psychiatry would later formulate as distinct disease entities" (Horwitz 2003: 41).

Further, dynamic psychiatry is not concerned with the treatment of symptoms, since symptoms are merely symbolic expressions of internal conflicts that involve an individual's entire personality, not an aspect of the makeup of their brain. In the Freudian model, there is no direct link between symptoms and diseases, and the same symptom can take on entirely different significance for different people. In this way, particular biographies of patients are more important for the meaning of symptoms than trying to make a connection to an underlying disease entity in an individual's brain, as is the case with biological psychiatry. Indeed, because the theory of repression, according to Freud himself, was the "cornerstone on which the whole structure of psychoanalysis rests", symptoms of illness more often than not masked the underlying cause that gave rise to them.

Only when symptoms can be categorized as expressing underlying diseases can a diagnostic, biologically-based system of psychiatry become possible. However, when symptoms are merely expressions of underlying conflicts within individuals which have highly variable meanings from person to person depending on their particular biography, they cannot be used to construct a well-defined diagnostic system. Dynamic and diagnostic psychiatry are opposed to each other. When calls for psychiatry to become 
more "scientific", more rigorous in its approach, grew in the 70's through the increasing popularity and dominance of the anti-psychiatry movement, diagnostic psychiatry took the reigns of psychiatric control from dynamic psychiatry, leaving it behind in its lack of scientific thoroughness. This swift and sudden paradigm shift which changed the landscape of psychiatry in less than 10 years was the second revolution in psychiatry.

In contrast to the dynamic model, diagnostic psychiatry defines diseases through the presence of overt symptoms, regardless of the causes of these symptoms. It perceives metal illnesses as natural entities that exist in the body and that generate the particular symptoms a person displays (Horwitz 2003). These are the assumptions of the NeoKraepelin school of psychiatry and the assumptions embedded in the DSM III.

The leading psychiatric classifier of mental illness who formed discrete mental disorders not reducible to one another was Emil Kraepelin, a German psychiatrist born the same year as Freud. However, during his career Kraepelin only spoke of two major diagnostic categories: Dementia Praecox, or what we know today as schizophrenia; and Affective Psychoses, what today would be referred to more specifically as Depression and Bi-polar disorder. However, his method of "scientific", symptom-based mental illness classification, in light of dynamic psychiatry's enlargement of possible pathological disorders, would lead to the creation and consolidation of the vast array of questionable mental illnesses individually listed as distinct pathologies in the DSM III and DSM IV.

Kraepelin's main contribution to psychiatry was his claim that there must be a specific brain or other biological pathology underlying each major psychiatric disorder. Emil Kraepelin attempted to combine the mental disorders identified by nineteenth 
century psychiatrists by grouping diseases together based on the classification of common patterns of symptoms, rather than by the similarity of major symptoms, as was the case during his time. One of the main principles of his method of classification was the recognition that any given symptom may appear in virtually every mental disorder; i.e. there is virtually no single symptom occurring in schizophrenia that cannot sometimes be found in manic-depression. In the absence of a direct physiological cause for each disease, it was only possible to distinguish mentall illnesses by their specific pattern of symptoms. For Kraepelin, what distinguished each disease was not any particular symptom or symptoms, but a specific pattern of symptoms.

In addition to his distinction between dementia praecox and manic-depression, Kraepelin is often credited with being the founder of modern scientific psychiatry, psychopharmacology and psychiatric genetics (Shorter 1997: 100). Though Kraepelin's contribution was largely ignored through most of the twentieth century due to the adoption of Freudian ideas, Kraepelin's basic concepts now dominate psychiatric research and academic psychiatry. Today, psychiatry is overwhelmingly biological and genetic in its orientation. Kraepelin's fundamental theories on the etiology and diagnosis of psychiatric disorders form the basis of all major diagnostic systems in use today, especially the American Psychiatric Association's DSM and the World Health Organization's ICD system.

In the 1970 s, psychiatry imported the diagnostic, descriptive model common to medical perception, replacing the dynamic model in its quest to be more scientifically rigorous. While diagnosis had little, if any role to play in the treatment of mental disorders in dynamic psychiatry, diagnosis became the bedrock of all psychiatric practice 
with the emergence of the diagnostic system. With this change, a catalogue of symptoms reflecting underlying mental disorders needed to be constructed: The DSM III. Whereas DSM I and DSM II had been based on dynamic, Freudian psychiatry and were meant solely as guides to broad underlying dynamic conditions or as reactions to difficult life problems and provided no elaborate classification scheme, DSM III had no room for the variability of individual biography in the schema of diagnosis. Indeed, by the time of the publication of DSM IV in 1997, the APA had dropped the term "neurosis", the "cornerstone of psychoanalysis", altogether from its psychiatric vocabulary. Diagnosis of mental illness had to be the same across time and place, and most importantly between psychiatrists. What was perceived to be manic depression by one psychiatrist had to be manic depression for another. More importantly, manic depression was manic depression regardless of an individual's biography, their life experiences, or any social or environmental cause of the symptoms classified as manic depression. The creation of discrete, medically treatable, biologically-based disease entities with verifiable symptoms - the basis of diagnostic psychiatry and the DSM III - left out, according to dynamic psychiatry, the most important aspect of mental disorders: the individual and their biography. As Jerome Wakefield writes: "The DSM is much more than a list of mental disorders. It is an attempt to use symptom lists to create necessary and sufficient rules for diagnosing all major mental disorders" (Wakefield 1999: 37).

According to critics of the DSM, who have tried to account for its sudden rise in popularity, a changing medical culture, along with social, political and economic factors, contributed to the decline of dynamic psychiatry and the ascendancy of the diagnostic system. David Horwitz writes that the emergence of diagnostic psychiatry can be 
modeled according to the Kuhnian model of a scientific revolution. In Kuhn's view, scientific models of thought - paradigms - are rooted in the social practices of scientific communities. The communities perpetuate and legitimate certain basic, fundamental assumptions about the nature, cause and control of the scientific discipline's subject matter. In so doing, the scientific community must necessarily reject alternative, competing models of the reality it claims to interpret. It is very rarely, if ever, the case that new paradigms are built upon older paradigms through an accumulation of more "accurate" information or knowledge which more closely reveals truth. The transition to a new paradigm is only undertaken so as to resolve a state of crisis in the previously dominant paradigm. The new paradigm will succeed only if it is better able to uphold a more convincing model of the phenomenon being studied within the relevant discipline, not because it has more accurately revealed the reality it studies. As David Horwitz writes:

The emergence of diagnostic psychiatry was not a gradual and piecemeal evolution, but a total reorientation of the discipline over a short period of time. The transformation of psychiatric thinking was less the result of new knowledge than an epistemological revolution that focused a new mental lens on the same set of psychological conditions... the demise of dynamic psychiatry did not result from the demonstration that its premises were false. Rather, these premises were no longer useful in a changed medical, social, cultural, political, and economic environment (Horwitz 2003: 54-55).

In the United States, dynamic psychiatry's legitimacy rested on its identification with the profession of medicine (Shorter 1997, Horwitz 2003, Kirk and Kutchins 1992). From the 1920 s to the 1970 s, dynamic psychiatrists in America legitimated their system of knowledge by considering themselves physicians (though Freud himself claimed that psychoanalysis need not be limited to medically trained doctors). This self-imposed 
status classification allowed dynamic psychiatrists to distinguish themselves from competing, non-medical health professionals such as social workers and psychologists. However, the dynamic model began to fall apart and to lose legitimacy in the eyes of the scientific community for not being scientific enough. Dynamic psychiatry's central method of treatment - the case history of the individual patient - was considered to be a respectable part of its medical practice until the late 1960s, when it began to be seen as unscientific. The main argument directed at the case study was that it was not verifiable, not subject to what Karl Popper had called "falsificationism". In the scientific community, the case study was seen as self-fulfilling, and unable to be transferred across psychiatric patients. Of course, this was the whole point of psychoanalysis, but the new scientific paradigm was not interested. The newly emerging medical norms emphasized statistical knowledge over the clinical intuition on which psychoanalysis rested, as well as the study of groups of patients rather than individual patients. Increasingly, the dynamic conception of what constituted a mental disorder was no longer compatible with the precise classificatory schemes that were beginning to dominate medical culture. As Horwitz writes: "The reason dynamic psychiatry was discredited as non scientific was that it had linked itself so thoroughly with the culture and institutions of medicine... The ideal of medicine demanded measurement systems in which symptoms were direct indicators of underlying disease entities, precise classification systems and clear criteria of therapeutic effectiveness (Horwitz 2003: 60). A psychiatric model based upon discrete disease entity creation provided the only intellectually respectable scheme for biologically and scientifically oriented psychiatrists. 
The rise of the anti-psychiatry movement in the US also contributed to dynamic psychiatry's diminishing popularity. While diagnostic psychiatry could protect itself against the anti-psychiatry movement by claiming that mental disorders were in fact not social constructs created by an oppressive scientific community upholding the status quo through the resocialization of its patients, but rather were natural brain disorders that could be corrected through the use of psychotherapeutic drugs, dynamic psychiatry was seen as an institution that stifled human freedom and autonomy. One of the best-known members of this group was Thomas Szasz. In 1961 he published what would become a manifesto of the anti-psychiatric movement: The Myth of Mental Illness. In his book Szasz questioned the very legitimacy of psychiatry itself (though he himself was a psychiatrist, a fact that no doubt legitimated his ability to speak in such a way upon the matter). Szasz claimed that psychiatrists were analogous to a modern clergy and that their claim to be medical professionals was little more than a charade, arguing that psychiatric concepts of health and disease were pseudoscientific value judgments (cited in Kirk and Kutchins 1991). No longer seen as a solution to repressive social institutions as it was in Freud's day, dynamic psychiatry itself began to be viewed as an institution that upheld conformity and quelled dissent.

Kirk and Kutchins retell the story of perhaps the most famous incident of the Anti-psychiatry movement. In 1973, David Rosenhan published his now (in)famous study "On Being Sane in Insane Places" in the journal Science. In his article, which has become a staple of first year sociology classes, Rosenhan described an experiment in which eight "pseudo-patients" were admitted into 12 psychiatric hospitals by falsely reporting a single symptom: each patient claimed that they heard an unclear voice that 
seemed to say such words as "empty", "hollow" and "thud". These symptoms had never been in any way connected to the historical diagnosis of what constituted schizophrenia. However, 11 of the 12 admissions to the psychiatric hospital were made upon the diagnosis of schizophrenia. The only interpretation of these symptoms not diagnosed as such was manic depression. The pseudo-patients acted out no other pathological behaviour. Details about their lives were truthfully conveyed, aside from their names and professions. Once admitted, the patients acted completely normally and stopped pretending to hear voices. Interestingly enough, the only people who seemed to catch on to this experiment were other patients. In total, the pseudo-patients remained in the hospital for an average of 19 days.

Upon being released, each patient was given the diagnosis of "schizophrenia in remission". Rosenhan used the results to espouse the validity of labeling theory, emphasizing the idea that psychiatric diagnosis will continue to be applied to the patient even if it is no longer warranted. Rosenhan's conclusion was that psychiatric diagnosis was unreliable and could have potentially negative stigmatizing consequences.

The problem of reliability became fundamental in the reconstitution of psychiatric knowledge and practice consolidated with the publishing of DSM III. Around the same time as Rosenhan's study began to make waves across the medical profession, the American Psychiatric Association had hired a man by the name of Robert Spitzer to begin revising the DSM III. Spitzer, trained as a physician, psychiatrist and psychoanalyst, was a self-proclaimed Neo-Kraepelin who had worked on the DSM II. Spitzer was also a professor of Psychiatry at Columbia University and Chief of the Biometrics Research Department at the New York State Psychiatric Institute. Spitzer 
began his career in the 1960 s by developing structured interview schedules for the assessment of mental disorder, including the Mental Status Schedule and the Psychiatric Status Schedule. At the time, these schedules were already considered a departure from Freudian models of mental illness in their distinct grouping together of symptoms of metal illness. In the 1970s, as part of a US National Institute of Mental Health-sponsored collaborative program on the Psychobiology of Depressive Disorders, Spitzer led in the development of more schedules: the Schedule for Affective Disorders and Schizophrenia (SADS), and the Research Diagnostic Criteria (RDC), all characterized by symptombased diagnostic criteria of mental disorder. As Kirk and Kutchins explain in their critique of modern psychiatry The Selling of DSM (1992), the anti-psychiatry movement's attack on the claims of reliability and validity by psychiatric practice put Spitzer in the difficult position of having to become the official defender of psychiatric diagnosis while at the same time reconstructing what actually constituted reliable psychiatric diagnosis. Spitzer took aim at Rosenhan's methods, claiming that they were merely examples of "logic in remission". Collecting his own data on discharged patients from hospitals, Spitzer concluded that patients were almost never diagnosed as being "in remission" at the time of their discharge. In fact, Spitzer claimed that these patients were almost always found still to be suffering from the disorder. This finding, according to Spitzer, proved that psychiatrists could in fact distinguish the sane from the insane.

However, Spitzer would conclude that psychiatry was perhaps not as reliable as it could be - something he was beginning to devote his attention towards fixing in the DSM III. Prophesizing the changes that were to occur from his revision of the DSM, Spitzer wrote in 1975 that "if psychiatric diagnosis is not quite as bad as Rosenhan would 
have us believe, that does not mean that it is all that good" (Spitzer 1975: 464). What is the reliability of psychiatric diagnosis? A review of the major studies of the reliability of psychiatric diagnosis prior to 1972 (conducted by Spitzer himself) revealed that "reliability is only satisfactory for three categories... reliability is no better than fair for psychosis and schizophrenia and is poor for the remaining categories" (Spitzer 1975: 460). Obviously, the question of reliability within psychiatry was a problem needing correction. Psychiatry itself needed to be medicalized, its variables of mental illness sufficiently operationalized to be reliable across time and space. Spitzer went on to write: "Recognition of the serious problems of psychiatric diagnosis has resulted in a new approach to psychiatric diagnosis, the use of specific inclusion and exclusion criteria, as contrasted with the usually vague and ill-defined general descriptions found in the psychiatric literature and in the psychiatric glossary of the APA" (Spitzer 1975: 451). Spitzer would lead the movement in the coming revolution in psychiatry that was gaining momentum. Yet, amongst all his talk about reliability, Spitzer did not discuss aspects of what constitutes validity of mental illness classification.

In the DSM II, psychiatric nosology did not provide a rigorous conceptual definition of mental disorder. Because classification is a form of measurement, a way of organizing the reality of objects, the question of validity should conceivably be fundamental within all classificatory models. However, in the medical construction of disorders and its elaborate classification and sub-classification of illness and disease, not enough critical examination of what these classifications and constructions are actually doing, what assumptions these models are based upon, is undertaken. As Kirk and Kutchins write: "Construct validity is distinguished from other types of validity... in that 
it concerns basic questions about the nature of reality. Having an operational procedure for determining whether a phenomenon belongs in a class does not fully address the question of what that construct or class is" (Kirk and Kutchins 1991: 29). For example, in the DSM II, homosexuality was defined as a mental disorder, and the claim was that it was a variant and pathological form of sexual development. In a telling example of Bowker and Star's idea that the process of classification always involves some sort of negotiation, at the 1970 annual convention of the American Psychiatric Association in San Francisco, gay activists began picketing conference events in order to have the APA's classification of homosexuality as a disorder removed from the DSM. Many members of the APA claimed that they had "scientific evidence" that homosexuality was in fact pathological and that any concession to gay activists to remove it from the DSM would be an unjustified political accommodation. However, other psychiatrists claimed this wasn't the case. Quickly it became apparent that the APA was unable to determine whether homosexual sex and the practices that it was made up of were valid grounds for diagnosing it as a mental disorder. In the case of homosexuality, psychiatrists could reliably identify a homosexual through simply asking about sexual practices (what Foucault would call "revealing the truth" about oneself). However, they could not identify the validity of claiming that homosexuality was itself a form of pathology. In order to address whether or not homosexuality was indeed a form of mental illness, the definition of what mental illness actually was and what it included had to be more convincingly operationalized. In order to combat arguments about the problem of validity, the APA altered the validity problem into one of simple reliability, implying that making diagnosis more reliable between psychiatrists was itself a measure of validity. 
By its very nature, the psychiatric community in its overwhelming need to totalize the human subject as something, whether it was homosexual or heterosexual, mentally ill or mentally healthy, found itself unable to look beyond the limitations its ontology had placed before it. The horizon of psychiatry's observable domain lay in its claims of reliability.

According to Spitzer, the reliability of the diagnostic system of psychiatry refers to "the extent to which users can agree on diagnosis applied to a series of cases" (cited in Kirk and Kutchins 1991: 133). The advantage of focusing so completely on the reliability of psychiatric diagnosis is that the problem of what constitutes a valid definition of mental illness can be downplayed or ignored altogether. Problems of reliability are reduced to methods and techniques on psychiatric decision-making.

The two common problems with psychiatric practice leading to differential diagnosis of the same sets of symptoms in a patient can be grouped into two categories: One, information variance occurs because psychiatrists often accumulate different information about their patients, thus often leading to different diagnostic conclusions as to what their patient's illness may be. The second category, criterion variance, refers to which pieces of information (even if information variance could be controlled or eliminated completely) are deemed to be the most important in making a diagnosis (Kirk and Kutchins 1991: 133). Whereas information variance has to do with the collection of information, criterion variance refers to the interpretation and categorization of information (Kirk and Kutchins 1991). According to Kirk and Kutchins, creating a completely reliable system of psychiatry free of information and criterion variance entailed the "control of discretion". Two strategies implemented by the emerging neo- 
Kaepelin movement in psychiatry to improve reliability were the control of the decisionmaking abilities of clinicians and, correlatively, altering the structure of the clinical interview. In general, these approaches, which gained widespread appeal and acceptance with the publishing of DSM III, were to structure the amount, nature and sequence of information that a psychiatrist would gather on a patient. Criterion variance was resolved by the creation and listing of distinct disease entities classified according to symptoms in the DSM III, thereby limiting the diagnostician's individual discretion as to the nature and cause of disorders. Information variance was solved by the structured interview schedule that standardized the information psychiatrists could gather about their patients in order to make diagnoses. "The development of highly structured interview protocols was the technical solution to the problem of clinicians conducting assessment interviews in their own individual ways and producing diagnostic unreliability." (Kirk and Kutchins 1991: 53) There is now a wide array of interview protocols for different classes of illness: The schedule of Affective Disorders and Schizophrenia (which comes in three versions to measure either current, lifetime or changed mental state); the Diagnostic Interview; the Renard Diagnostic Interview and the Structured Clinical Interview. What these interview schedules have in common is a pre-arranged progression of questions the responses of which are subsequently matched with the diagnostic criteria of the DSM.

This control and filtering of what information is gathered and how it is to be interpreted by psychiatrists prevented them from expressing individual and autonomous clinical judgment when making a diagnosis of mental disorder and options for treatment. More fundamentally, this system ignored individual patients' biography and their autonomy to interpret their own relationship to their sickness. As Ivan Illich (1975) 
conveys, the medical profession takes away the will of people to suffer their individual reality and come to a greater understanding of themselves and their human condition. Further, the diagnostic criteria and interview schedules allow for little, if any meaningful dialogue between patient and psychiatrist. While it is true, in Foucauldian terms, that dynamic psychiatry is a form of government whereby patients' conduct is conducted by the bio-power inherent within the medical community paradigm, it is no less a form of government (indeed a more ominous form of such) when there is no chance of even contributing to the understanding of one's illness. The problems that arise from the need to have a perfectly reliable, scientific psychiatric method - one that eliminates criterion and information variance - become ways of viewing patients, thereby dictating how mental illness is perceived as well as how it is treated. DSM III, in its quest for reliability, validates a plethora of mental illnesses in the psychiatric community while never really questioning their validity. Since patients do not have individuality or personal history within diagnostic psychiatry, nor any ability for the interpretation of the causes of their disorder or its treatment, psychotherapeutic drug intervention is a logical next step in the treatment and normalization of patients.

\section{Making Up People}

In order to understand the constitution and normalization of psychiatric patients, Foucault's employment of what he calls "bio-power" - power over life and its processes that, once constituted as knowledge becomes a form of normalization of life itself, is an intriguing model to follow. While not focusing on the political aspects of bio-power, with the creation of so many various disorders and the correlative creation of standardized knowledge about disorders and how to treat them, it is my view that the 
medical community perpetuates bio-power through individuals and their bodies. Biopower employs the technologies of self found within psychotherapeutic drugs to normalize patients. Patients come to see themselves in terms of health and wellness. The standard of their lives is dictated by the "amount" of health they have stored up within them, or is somehow expressed more completely through the intervention of medication. While I will discuss further the impacts of bio-power and the commodification of health in the next chapter, a variant of bio-power sketched by Ian Hacking termed "dynamic nominalism" highlights the implications for human conduct and motivation in light of the diagnostic classification of mental illness into distinct entities.

Hacking's dynamic nominalism (or "making up people") simply claims that as new modes of description about human behavior, or in our case mental disorder, arise, so to do new possibilities for action. In this theory, Hacking combines labeling theory, which holds that social reality is conditioned and created by the labels applied to people, their actions, institutions and communities, with a Foucauldian understanding of discourse and the constitution of subjects. As Foucault himself famously said of the intentions of his work: "We should try to discover how it is that subjects are gradually, progressively, really and materially constituted through a multiplicity of organisms, forces, energies, materials, desires, thoughts etc." (Foucault 1980: 97). Hacking claims that various kinds of labels interact with the very things that they classify and in so doing the classification itself may be modified or even replaced after a period of time (which is an integral aspect of all classification systems according to Bowker and Star). Further, classifications do not just interact with the classified because the people so classified internalize the classification. Instead, the classification changes because it interacts with 
those who are so described through and within institutions and practices that are themselves predicated upon such classification.

Dynamic nominalism is a method to understand the dynamic interplay of description and the possibilities for action it presupposes, the ways in which the labeled act out their label, and at the same time, come to contribute to the evolution of ideas about themselves. Hacking writes: "The claim of dynamic nominalism is not that there was a kind of person who came increasingly to be recognized by bureaucrats or students of human nature, but rather that a kind of person came into being at the same time as the kind itself was being invented" (Hacking 1983: 228).

It is my view that the current system of psychiatry, with its standardized ways of viewing, labeling and treating individual patients, is a system of technologies creating, mobilizing and expanding mental illness and reconstructing individual subjectivity. By creating and legitimizing a system of distinct disease categories according to the grouping of symptoms, while ignoring personal history and social factors, and while not recognizing the patient's ability to interpret their own affliction in an autonomous way, diagnostic psychiatry creates and imposes a type of subjectivity centered around the idea of medical well-being.

The dynamic interplay of categories and the categorized is evident in the DSM's continual need to be updated $(1952,1968,1980,1987,1994,2000)$. Around once a decade, subjects who have come spontaneously to fit the categories given to them by their psychiatrists, physicians, or even themselves through the help of pharmaceutical industry advertising (as will be discussed in chapter three), contribute to an increase in medical "knowledge" about their supposed affliction(s). "Keeping up" with the ways in 
which patients fill the roles they are given alters the institutional psychiatric knowledge about these people and their disorders. Correlatively, the new knowledge becomes standardized in updated editions of the DSM, each time adding to the total number of disorders listed, as well as the amount of written text attempting to explain these disorders. In such a system, mental illness creation and expansion could presumably continue indefinitely as categories and the categorized mutually alter one another until perhaps the idea of what could ever be recognized as "health" fades into the obscurity created by the plethora of disorders.

The point in mobilizing the theory of dynamic nominalism is to highlight the ways in which the classifications found within the various editions of the DSM may alter the way people experience themselves. This occurs not just because actors may come to act out their labels, but also through the stigmatizing perception of those around them who view them as their classification, acting toward them accordingly and reaffirming their experience of the illness. This stigmatization is both institutional and social. Classification stigma becomes embodied in institutions, correlatively perpetuating the prevalence of disease - of course this is most obvious within the institution of psychiatry, but it is also present in the various patient advocacy groups who reify the concept of mental illness in their very attempt to break down its stigma.

In Hacking's account, the practice of "making up people" consists of two vectors of analysis: classificatory looping and bio-looping. Classificatory looping is the process described above, whereby classes and the classified interact in ways that create an ever shifting ground of classification. With this, Hacking proposes a related aspect of this process called bio-looping, whereby changes in our ideas about ourselves may even 
change our physiological states, such as occurs during the practice of yoga. The types of looping are mutually reinforcing. Bio-looping may even be the beginning of an explanation of the placebo effect that has puzzled so many in the health community. The full implications of bio-looping, if there is any truth to it, are profound. Of course, setting out to prove this theory is beyond the scope of this thesis. I only wish to propose the possibility of such an occurrence as expressed through the creation of diagnostic psychiatry. Further, it is clear that such a theory is not absolute or consistent across time and place or that it could be applied in the same way to different people. No doubt this is why Hacking claims that there is not a general story to be told about making up people; each category has its own history and its own resistance. Using Hacking's framework, I have only explored one vector of his model - the vector of labeling from above by the psychiatric community which creates a reality people may eventually come to make their own and to see themselves through. The other vector which I am ignoring (at the risk of incompletion) is the vector of autonomous behaviour of labeled individuals. However, the point should still be clear: who we are as individuals is not simply a matter of what we have done or find ourselves doing in the present; who we are is also a product of what we are able to do in the future. As Hacking writes: "making up people changes the space of possibilities for personhood." This claim will be discussed further in chapter four.

\section{What is Mental IIIness?}

Can there ever be a valid definition of what constitutes criteria of mental illness? Can this definition be valid and reliable at the same time? Are definitions of mental illness simply creations of the medical community? Where do the boundaries lie, if there are any at all, between what constitutes "normal" and "abnormal" mental health? 
Most mental health professionals, as well as the populace at large, assume the disorders listed in the DSM are natural entities and not arbitrary constructions. In the DSM, mental disorder,

is conceptualized as a clinically significant behavioral or psychological syndrome or pattern that occurs in an individual and that is associated with present distress (a painful symptom) or disability (impairment in one or more important areas of functioning) or with significantly increased risk of suffering death, pain, disability, or an important loss of freedom. In addition, this syndrome or pattern must not be merely an expectable response to a particular event, e.g., the death of a loved one. Whatever its original cause, it must be considered a manifestation of a behavioral, psychological or biological dysfunction in the person. Neither deviant behaviour, e.g., political, religious, or sexual, nor conflicts that are primarily between the individual and society are mental disorders unless the deviance or conflict is a symptom of a dysfunction in the person. (APA 1980: 6)

However, what at first appears to be a fairly unproblematic definition within the DSM's introduction, becomes problematic in the analyses of the disorders within it. The DSM goes on to state that there is no assumption that each mental disorder is a discrete entity with sharp boundaries between it and other mental disorders, or between it and no mental disorder. However, as it has been shown, this is not the case for self-proclaimed NeoKraepelin psychiatrists. The definition above was written primarily in response to antipsychiatric accusations that claimed coherent and valid distinctions could not be drawn between actual disorders and non-disorders. The DSM attempted to use the concept of disorder in a way consistent with its use in the medical sciences generally. The DSM's definition of mental disorder makes distinctive statements about what disorder consists in. Disorder is to be found within individuals and is not a product of an unfortunate event in one's life; and disorder is the result of the way an internal mechanism normally functions which can lead to some sort of conflict between the individual and society. 
However, according to critics of the DSM such as David Horwitz and Jerome Wakefield, the DSM list of disorders fails to live up to its own conceptual standard.

Mental disorders should always be distinguished from the expected psychological consequences of stressful conditions, as stated in the DSM itself. But with a psychiatric method that cares little for patients' personal autobiography, indeed a method that does not even have a method for retrieving it, as is shown through the creation of standardized psychiatric interview schedules, many disorders that arise in response to social stressors are in fact included within the DSM. And upon this basis many everyday, quite normal reactions to everyday reality are pathologised as natural brain dysfunctions in need of correction with personality and mood altering drugs. For example, this fact is evident with the spread of "generalized anxiety disorder" throughout the US after September 11, 2001. What was thought to be a relatively minor disorder even in the revised DSM III in 1987 , affecting $1.8 \%$ of the US population, quickly became close to an epidemic after September 11, with drug companies and psychiatrists claiming that GAD effected close to $14 \%$ of the population (Greenberg 2003: 3). This 15 year period is a very short time for so many Americans to have altered the very chemical makeup of their brains. But as David Horwitz writes: "Contrary to its general definition of mental disorder the DSM and much research that follows from it considers all symptoms, whether internal or not, as signs of disorder. The result is that contemporary psychiatry and psychiatric epidemiology considerably overestimate the amount of mental disorders" (Horwitz 2003: 37).

It has been shown that the need for reliability was at the base of the creation of diagnostic psychiatry's classification system itself, which in turn lessened the emphasis 
placed on validity. The best answer as to the question of what the validity of mental illness diagnosis could be comes from Jerome Wakefield, who tries to bridge the difficulties inherent in the diagnostic system of illness classification and the social constructionist approach. Wakefield attempts to reconcile the very difficult conceptual relationship between the biological and social contributors in the make up of mental disorder. Wakefield argues that a mental disorder should be perceived as a "harmful dysfunction": "harmful" a term based on social norms, and "dysfunction" a scientific term referring to the failure of a mental mechanism to perform a biological function. Therefore, according to Wakefield, a condition is a disorder if a) the condition causes some sort of harm to the individual as normalized by their culture and b) the condition results from the inability of some internal mechanism to perform its natural function within the brain. The concept of individual harm and internal dysfunctions overcomes the common misuse of disorder classification found in the general population as well as its exploitation and manipulation for the purposes of profit by the pharmaceutical industry (see chapter three).

A fundamental consequence of the diagnostic system of psychiatry is that it legitimates chemical intervention upon subjects. In this regard it is not so different from the reconstitution and technologies of the self present within the dynamic system. As Peter Kramer writes: "it is now sometimes possible to use medication to do what once only psychotherapy did - to reach into a person and alter a particular element of personality. In deciding whether to do so, the psychopharmacologist must rely on skills we ordinarily associate with psychotherapy" (Kramer 1997: 97). Further, because it does not remain consistent to its definition of mental disorder, common reactions to life events 
are made into sickness; the realm of sickness spreads deeper and deeper into the human condition itself. Life is meaningless and personality can be bought in the form of a pill. Such is the historical junction in which we find ourselves.

In the next chapter, I turn the origin of "real" mental disorders. Within a society concerned with the creation and manipulation of human desire, individuals are increasingly disconnected from their ability to interpret emotional responses to external stimuli in any productive way. Emotion, as the optic through which individuals achieve self-understanding and the ability to direct the course of their life as well as being the very thing that connects individuals to the external world, has been "unhitched" from its biological function of orienting individuals within their environment. The result is a culture of narcissism, where the self cannot differentiate itself from the world it is a part. 


\section{Chapter Two}

In the previous chapter the thesis was put forward that the emergence of the diagnostic system of psychiatry led to the expansion and proliferation of categories of mental illness. In the overtaking of Freudian ideas of pathology and the corresponding creation of a system of distinct diseases characterized by the grouping of symptoms that allowed for reliable diagnosis across time and place, this new psychiatry ended up pathologising aspects of the human condition itself - the human need to feel pain, anxiety and loneliness and to come to self-understanding through it. Further, it was shown how actors themselves could, in principle, come to act out the diagnostic category they were given at the same time as the medical community itself changed the knowledge about these disorders. The implication of these processes is "diagnostic bracket creep" into grey areas of what distinguishes health from ill health.

However, it is not completely accurate to assume that the proliferation of mental disorder is solely dependent upon the medical community's innate need for disease creation. I believe there is another aspect to this story feeding into and reciprocally conditioning the use and spread of psychotherapeutic drugs. There are such things as "real" illnesses. Illnesses - whether mental or physical - are not solely creations of the medical community. There are forces within society propelling the expansion of illness as well as a nihilistic groundlessness that cannot come to any kind of judgment as to the consequences of such a practice. Indeed, as Ivan Illich has written: "In every society, the classification of disease mirrors social organization" (Illich 1976: 43). While social theory has pulled back somewhat from believing we are now within a completely "postmodern" and relativist world sewn by nothing more than power relations, it is the 
thesis of this chapter that essential human needs - the need to eat, have shelter, and relatedness with others - and their fulfillment compose the fundamental attribute needed for "real" health. And while it is at the same time true that how health is classified and how it is managed by the medical community specifically often gets formulated and reformulated throughout history, it is important to understand that health in this sense is a political measurement, not simply a medical one. The various reformulations of what constitutes ill health change with the political organization of society itself, but the essence of what is being reconfigured remains the same. Health, as nothing more than a measure of the capacity of individuals to cope with their internal states and their environmental conditions, is itself a political phenomenon and as such gets reconfigured in response to political forces dictating the allocation of social goods needed for health (which is not exclusive to medicines but includes such things as equitable access to safe water and food etc., as well as the length of the working day and labour conditions). For example, traditional cultures confronted pain, ill-health and death as challenges to be confronted and responded to by both individuals and the community. In our "medical civilization" these social realities are turned into problems to be managed or produced out of existence by large bureaucratic structures which come to only perpetuate them (Illich 1977: 133). The implication for my own discussion is that the current reconfiguration of mental health revolves around the intervention and normalization of emotion particularly mood.

The "essence" of a human being (in the sense that a human being is made up of certain constitutive properties that, if removed, would no longer leave a human being - a rather mundane form of "essentialism") is to be a culturally bound, value positing being. 
In a culture consumed with the creation and manipulation of needs such as ours, needs become a value upheld by this culture itself. This mass creation of desire, of need in society, leads to an individual inability to control emotion which could be said to be the beginnings of mental illness. Anxiety, mass depression, and loneliness are products of a society manipulating one of the very things that connects individuals to the society of which they are part: emotion.

Emotion is integral to selfhood. From a phenomenological point of view, "an individual's feelings of distress, anxiety, alienation and love are simply an expression of the personal and private apprehensions the individual has made of the world. As such, emotions are emblematic of the individual's understanding of self, others and their social milieu" (Finkelstein 1980: 119). Emotion, in this sense, can be thought of as an individual's interpretation of bodily sensations arising from their experience in the world. Emotion is not a sensation, or a chemical reaction in our brain as is assumed by biological psychiatry, but a "relational, inter-subjective phenomenon which joins us to others and is produced via our interactions with others" (Lupton 1998: 22). People are their emotions. Emotions provide "situatedness" within the world as well as a means through which self-understanding can take place. As sociologist Norman Denzin wrote: "emotions are not things, they are processes. What is managed in an emotional experience is not an emotion but the self in the feeling that is being felt" (Denzin 1984: $50)$.

Mass consumerism and needs creation places the fulfillment of human needs beyond the ability of individual control, giving rise to a narcissistic culture whereby the demarcation of where the emotional self of an individual begins and the society of which 
they are a part, where they are conditioned and brought up, ends. Culture, being an organizational technique creating value and meaning for the human beings within it, when concerned with the creation and temporary fulfillment of needs, creates and upholds a personality type concerned almost exclusively with immediate emotional gratification. Because emotion is what connects individuals and society (Lupton 1998), its manipulation and medical intervention necessarily implies cultural narcissism. The culture of narcissism loses touch with its own health, for it has no means of gauging what it is.

\section{The Critique of Health}

In the late 1970s, Ivan Illich published Limits to Medicine, arguing that professional medicine itself was a threat to health. The very thing the health profession, with its system of practices and corresponding ways of viewing the human body, proclaimed to produce, namely healthy individuals living in healthy societies, it unknowingly negated. Doctors, hospitals, clinics, pharmacists and the rationality that bound them together were all considered attributes of a system that was contributing to the decline in the health of individuals and communities. And this decline in health was more than a simple matter of malpractice. Modern medicine, in Illich's view, was a threat to health in that it took away an individual's ability to suffer pain in a personal and autonomous way. This phenomenon he called "iatrogenesis", derived from the Greek "iatros", or physician, and genesis, meaning origin. Iatrogenesis was such a threat to health that Illich had no qualms with labeling it a modern epidemic. Illich broke iatrogenesis into three subgroups: clinical, social and cultural. 
For Illich, clinical iatrogenesis is as old as medicine itself and is the very phenomenon that leads to the creation of medical illness in the first place. Clinical iatrogenesis includes all conditions for which remedies, physicians and hospitals are themselves the pathogens which led to the creation of disease. Illich cleverly introduced the phrase "disabling non-diseases" to highlight the result of the medical treatment of non-existent diseases which he saw as increasing indefinitely. With the commodification of health itself through the creation of publicly and privately administered health care plans, iatrogenesis becomes a social phenomenon whereby the social control of the population by the medical system turns into an economic activity in itself. Health is no longer something inside individuals, maintained through their own autonomy, but is bought on the medical market or acquired through the paid consultation of physicians or psychiatrists.

Social iatrogenesis ends up legitimizing social arrangements and pathologising social breakdown as expressed through individuals and their health. And as I have shown, this is what is happening with the intersection of bio-power and nihilism. Psychotherapeutic drug use is an expression of the needs of a widespread narcissistic personality to absorb the constant flux of consumer desires being created throughout society. This in turn leads to the nihilistic view that there is no value within life itself as it is bought and sold and paid for. The medical community pathologises the effects of this as expressed through mental health. Psychotherapeutic drug use, however, instead of combating the social effects of anxiety and depression due to a perceived meaninglessness in life, actually contributes to this phenomenon, reinforcing and perpetuating it. 
Cultural iatrogenesis arises when the medical profession takes away from individuals the ability to suffer their own individual reality and to gain understanding about themselves through it. Suffering is deprived of its inherent meaning. With the management and annihilation of all pain in a society thoroughly medicalized, the demands placed on individuals which could lead to understanding of their existence within the world are turned into ignorance of their self and their health. Modern society detaches pain from any subjective context in order to annihilate it. Pain is to be avoided at all costs - even at the cost of the individual's sense of self. However, the extermination of pain is the extermination of what it means to be human; it is the death of humanity's unique ability to conceptualize itself and its body and to derive meaning from them. Self-healing is the most profound access to an understanding of being alive. What is referred to as the placebo effect in medicine is the term given to the medical community's inability to understand the ways in which humans come to deal autonomously with illness in personal ways, thus healing them without "conventional" medical intervention.

Critical anthropologist, Stanley Diamond has written that, "the mental health establishment is a pathological symptom of the society that created it; it is part of a fragmented social process within which the alienated study the alienated... the conventions of our civilization conceal our social fragmentation while suppressing the insights that might conceivably be generated by its exposure" (Diamond 1974: 230). Psychotherapeutic drug use is the latest medical technology seeking the minimization of individual selves and individual understanding of the articulation of self in modern society. Because health is simply the extent to which individuals cope with and attune 
themselves to the environment they are in, the health of a society as a whole is dependent upon the insights and adaptations individuals, as microcosms of society, contribute to their own health, and thus to the health of the society as a whole. This cannot take place through the bureaucratization or privatization of health. Ill-health could be said to be an expression of contradictions within society that are expressing themselves through individuals and their health. Medicalization of human emotion becomes a political technology to quell individual protest as expressed through mental health. Instead of treating the cause, or letting individuals play out in a productive (or destructive) way the illness within them, the medical community resorts, for fear of social collapse, to the relieving of symptoms instead of fixing their cause.

The narcissistic personality within modern society, which is an articulation of social contradictions playing themselves out through individuals, is most disturbingly seen in what we know as schizophrenia. Schizophrenia is commonly referred to as a "biochemical brain disorder." The symptoms of the illness include disorganized thinking, delusions, hallucinations, and sudden changes in emotions and behaviour. According to Health Canada, schizophrenia will affect one percent of the population and it is estimated that 300,000 people in Canada will be diagnosed with the disease at some point in their lives (Health Canada: Report on Mental Illness in Canada, 2003). In the US, the National Institute of Mental Health (NIMH) estimates that approximately 2.2 million American adults, or about 1.1 percent of the population age 18 and older, have schizophrenia. However, despite what the medical community lists as the causes of schizophrenia, schizophrenia can also be viewed as the most complete and totalized form of narcissism, where there is no separation of self from society within the mind at all, 
only mixed and flickering images that come and go at random - the very products of consumer society. With schizophrenia, the self becomes a reflection of the discontinuity in the socializing structures expressed through the personality of individuals within society. As Diamond writes: "schizophrenia, as we know it, gives every indication of being a protest against and a response to the problem of learning how to be human in contemporary society" (Diamond 1974: 239). Schizophrenia is the end logic of modern existence, its most complete articulation through the individual.

The conservative cultural critic Christopher Lasch once wrote that "in a time of troubles, everyday life becomes an exercise in survival" (Lasch 1984: 15). Lasch's point, that modern society has lost any meaningful connection with the authority and teachings of the past which could guide and nurture human community in the present, appears to hold true today given the statistics I mentioned in the introduction, even if in actuality the number of people estimated to have a mental disorder depends to a large extent upon ways of classifying disorder and the means of measuring disorder. The narcissistic personality that Lasch ascribes to modern North Americans, in which the differentiation between the self and the world in which it is situated becomes so blurred that individuals become fixated on immediate gratification - trying to fill themselves up with disposable commodities, while in reality becoming more and more empty emotionally and psychologically - appears to be realizing itself through the increasing prescription and consumption of psychotherapeutic drugs. While narcissism itself is a psychiatric term commonly associated with Freud, it is useful, not so much as a theory that claims narcissists have an innate need to regress back to the comfort of the womb, but rather to elucidate the overwhelming difficulty individuals appear to be having in becoming 
individuals in a "society under siege" as Zygmunt Bauman put it (Bauman 2002). As part of the need to quell the difficulties and anxieties of modern existence - whether economic, social or interpersonal - psychotherapeutic drug use becomes a quick and painless alternative to dealing with reality. In so doing, the world "out there" becomes reconciled with one's emotional capacity to deal with it internally, blurring the boundaries between the self and the external environment's emotional effects upon the self. In this way, narcissism can be seen to be a defense against a world where individual integrity and autonomy cannot easily be reconciled with the demands of modern life. Life becomes emptied of meaning the more and more meaning itself becomes dislodged from actual experiential understanding. With no ability to orient oneself authentically in such a world, the ability to distinguish one's self from the society washing over and through it breaks down and is swept away. The result is narcissism.

So it is my contention that "real" disease and illness creation is also a result of a society unable to reconcile the emotional lives of its members with its own needs of reproduction as expressed through the fundamental driving force within it: mass consumerism. What is occurring is the intersection of two vectors of social reality - on the one hand bio-power pathologising various aspects of the human condition and intervening upon it through technologies of the self created by psychotherapeutic drugs, while at the same time, society itself is increasingly emptied of meaning and value for the individuals who compose it because of the break down of a solid distinction between the self and the world outside of it due to the flux of desire creation characteristic of modern day "hyper capitalism". Each vector reinforces the other. 
Lasch goes on to sum up what could be thought of as the most common explanation for the expansion of psychotherapeutic drug use created by the breakdown of the boundaries between self and society: "plagued by anxiety, depression, vague discontents, a sense of inner emptiness, the "psychological man" of the twentieth century seeks neither self-aggrandizement, nor spiritual transcendence but peace of mind, under conditions that increasingly militate against it." (Lasch 1979: 13) If culture is said to be a symbolic system which transforms physical reality into experienced reality (Lee 1986), the culture we live within is in breakdown, or in such a rapid state of change that the social demands of the individual are not as yet being balanced by organizational structures mediating these demands. No longer is there a sharp, clearly defined demarcation between individuals and the society of which they are a part. Whatever the modern status of the Freudian tenets upon which Lasch bases his assumptions, or the explicit appeal to a lost brand of Conservatism which believes that it is still somehow possible for the family to be a "haven in a heartless world", I believe Lasch is hitting upon something fundamental in modern society. This search for "peace of mind" begun in the $20^{\text {th }}$ century now, in the $21^{\text {st }}$ century, comes in the form of altering the mind's very composition through the intervention and manipulation of the brain's neural pathways the very pathways that allow for the expression of individuality, creativity and personality. While bio-power plays its part in the control of subjects, it effectively mobilizes discontent for the purposes of allowing this discontent to be governed and controlled through the creation of psychotherapeutic technologies of the self.

Psychotherapeutic drug use makes individuals emotionally disconnected from reality. In a culture of narcissism, an individual's sense of self is made as small and 
helpless as possible. Psychotherapeutic drugs "re-energise" humans, making functioning in a world with no solid ground from which to find meaning and permanence in their life more tolerable and easily achieved. Yet, if emotion - particularly mood - is a biological response to social stressors, a means for recognizing the need for individual social adaptation, and the society in which we live does not offer either the material or ideal tools for the individuals who compose it to be emotionally well-adjusted within it, psychotherapeutic drug intervention, which alters mood and personality in often socially appealing ways (making individuals more charismatic, energetic and socially competent), becomes a way for individuals to reconcile themselves to forces outside themselves that are causing the creation of moods potentially harmful to themselves and others. The creation of a-historical subjects, which is a product of modern psychiatry's push for reliability, as mentioned in the previous chapter, conditions and further produces a world seemingly without any meaning accessible to individuals.

In Lasch's view, our "minimal selfhood" is not simply a defense against perceived danger, against a fear of terrorism or maladjustment to the demands of modern existence, but is much deeper: our minimal selfhood is a "response to the replacement of a reliable world by a world of flickering images that make it harder and harder to distinguish reality from fantasy" (Lasch 1984: 19). Society is no longer a place to realize one's identity, to actualize one's individuality. In modern society, individuals are constantly forced to adapt to the demands of work and life. Individuals have not one fixed identity but many. And it would appear, with the creation of psychotherapeutic drugs, identity know comes in the form of a pill. No longer do people feel in control of their destiny, but (willingly or unwillingly) submit to needs that come from outside their 
control. Individuals within this culture of narcissism neither look back upon their lives as a source of knowledge to be mined for clues to negotiating their present; nor do they look ahead for fear of all that is yet to come. Individuals are stuck in a series of "nows" laid side by side both behind and front of them. As Bauman poetically put it, the consumer market "proffers eternity in installments, each bit coming ready for immediate use and meant to be disposed of without regret or remorse once it is used up." (Bauman 2002: 196). Caught within these circumstances, the self contracts into a defensive core incessantly battling against the demands of modern existence. Or as Hannah Arendt stated, the self is caught between "past and future", the past propelling individuals into a future they cannot comprehend at the same time as the future drives individuals back into the past (Arendt 1954). Thus, emotional equilibrium in modern society demands a self with as little authentic emotional connection to the world as possible.

Psychotherapeutic drug use not only minimizes the individual self, it necessarily obliterates the self, allowing for the reconstruction of mood and personality in accordance with social demands facing the individual. When social critics like Herbert Marcuse questioned modern advanced capitalist society's use of technology to create onedimensional culture and one-dimensional citizens unable to realize their individuality, little were they able to fathom the extent to which technology would come to alter the brains of the very people within technological society (questions regarding technology will be addressed in chapter four). This is a further expression and a deepening of the thesis of modern narcissism. This fact is also highlighted in Peter Kramer's book Listening to Prozac, which explores cases where his own patients have undergone such a complete change in their social and interpersonal lives while on Prozac that their own 
conception of self is so altered that Kramer is often told by patients coming off drugs like Prozac that they "no longer feel like themselves." These drugs allow for an imaginary sense of self amongst social conditions that allow little or no room for an authentic, unmedicated sense of self. However, this illusory self, achieved through psychotherapeutic drugs, is in fact a deepening of the loss of self.

Max Horkheimer, in his essay "the Concept of Man" wrote that the emerging movement of society, with its widespread mechanization, favours a mentality solely fixated on the now, leaving no room for memory or imagination. In a society dictated by the demands of commodity production and their correlative psychological effects, "the capacity for experience that transcends the immediate situation is being atrophied" (Horkheimer 1972: 167). Of course, Horkheimer is thinking of pre-World War Two industrial society. However, his thesis still holds in an era of automation and in a society dominated by the production of needs instead of actual products. Christopher Lasch writes in his Culture of Narcissism that "every society reproduces its culture - its norms, its underlying assumptions, its modes of organizing experience - in the individual, in the form of personality... The process of socialization... modifies human nature to conform to the prevailing social norms" (Lasch 1979: 34). Through individuals and their health a microcosm of society can be glimpsed. For modern society, these norms are reproduced with the help of psychotherapeutic drugs, which are increasingly engineered toward creating desirable socialization effects within the individuals who take them.

Consumption at our current level presupposes the creation of more and more needs, both economic and symbolic. This leads to a world based on insecurity and inability to quell anxiety. As Bauman writes: "uncertainty-generated anxiety is the very 
substance that makes the individualized society fertile for consumerist purposes... more often than not, the production of consumers means the production of 'new and improved' fears" (Bauman 2002: 199). For Bauman, what distinguishes modern consumption from previous modes consumption is its emancipation from the expressed social uses which had set its upper-bound and lower-bound limits. Today, consumption is a thing in itself, for itself. Previously, needs were fulfilled and the emotional tension created within individuals was dispersed through the act of consumption; now, however, needs are perpetually created and strengthened. Because of this, Bauman, along with many other social critics, no longer talks of needs production, but of desires production. In this way, individuals become more and more unfulfilled or see life as valueless because of the inherent nihilism which can only be the end result of such an outlook. Commodities are bought, consumed or hoarded in an attempt to acquire some kind of sense of self, but to no avail. Life in consumer society is based on the creation of needs, not their fulfillment. As Marx wrote back in 1844, "Each person speculates to create a new need in the other person, in order to force him into a new dependency, to a new form of pleasure, hence to his economic ruin... With a multitude of commodities grows the realm of alien things which enslave man" (quoted in Fromm, 1955: 135). The need for mood altering drugs, which attempts to give individuals "normalcy" is the result of this constantly shifting ground upon which individuals attempt to navigate their lives. Consumer society creates the need for psychotherapeutic drugs and also finds its fulfillment through their creation and widespread distribution.

Bauman continues: "Despite its successive and always short-lived reifications, desire is 'narcissistic': it has itself for its paramount object, and for that reason is bound 
to stay insatiable however tall the pile of other (physical or psychical) objects marking its past course may grow" (Bauman 2002: 184). Under conditions of modern consumption, Freud's pleasure and reality principles, thought by Freud himself as incompatible and mutually antagonistic, which give rise to repression and discontent, have been reconciled. According to Bauman, in consumer society pleasure itself is the essence of reality; fluidity the ultimate solidity of social life. Thus modern society becomes dependent upon the narcissistic personality. Lasch contributes to this view: "a culture organized around mass consumption encourages narcissism... not because it makes people grasping and self-assertive but because it makes them weak and dependent. It undermines their confidence in their capacity to understand and shape the world and provide for their own needs" (Lasch 1984: 33). The pathologisation of aspects of the human condition which are not compatible with this need, or are the results of an inability to adjust themselves to this social need (depression, social anxiety disorder, panic disorder), is more and more being corrected by psychotherapeutic drugs, thus easily integrating everyone into the flux of consumer desire so prevalent within society.

However, in the act of consumption individuals do not relate to the objects they consume, or find themselves surrounded by, in any human, concrete or aesthetic way. The question is not how many material possessions a person may have, but how meaningful the connection is to these possessions. In and of itself, having the choice of 12 different types of cappuccino does not innately enrich or degrade human existence. Consuming 12 different types of cappuccino for no other reason than the sake of doing so, however, leads to the degradation of human existence. This Erich Fromm posited, perhaps a touch superficially, as the differences in relatedness to the world in which 
individuals can choose to live: the Being mode, where objects as well as people are related to in an authentic way simply because they exist or are as such; and the Having mode, whereby an individual's relatedness to the world is characterized by ownership and possession for their own sake. As Fromm wrote close to 50 years ago:

Our way of consumption necessarily results in the fact that we are never satisfied, since it is not our real, concrete person which consumes a real concrete thing. We thus develop an ever increasing need for more things, for more consumption... Originally... consumption was a means to an end, that of happiness. It has now become an aim in itself... the act of buying and consuming has become a compulsive, irrational aim, because it is an end in itself, with little relation to the use of, or pleasure in the things bought and consumed. (Fromm 1955: 134-135)

For Fromm, social structure is reflected in the personality structure of society's members. Human beings struggling with self-actualization in a world that does not allow for self in the first place is reflected through the mental health of individuals. If the assumption is true that humans, as products of culture, are innately in need of self-reflection and also in need of having permanence and meaning in their lives, a society based on the negation of meaning and the perpetuation of meaninglessness results in the increasing emptiness of its members and thus to their "ill-health". What began as the highest value of capitalist production - work as an ascetic value in and of itself - was transformed into the Utilitarian attainment of the greatest happiness for the greatest number, and has now necessarily devalued itself into its opposite: the greatest emptiness for the greatest number. Work is a means to acquire money and the fetishism of commodities that results has given birth to an inhuman world where individuals and their sense of self are mediated through this fetishism. Our sense of self is itself a commodity to be bought and sold. However, this sense of self becomes obsolete and in need of continual replacement. 
This, however, conflicts with humanity's need for permanence, leading to an inability to distinguish reality from fantasy.

Of course, consumerism does indeed imply more than just economic consumption. However, I do not view postmodern conceptions of consumption as being a radical break from older, economic conceptions of consumption: The one necessarily leads to the other (the Baudrillardian theory of hyper-reality as being cut off from the control of capitalism's production impulse are overstated and far-fetched conceptualizations for our current cultural impasse, but this is not to discredit the idea that we are heading in such a direction). As Lasch suggests, the psychological effects of consumerism can only be understood when it is thought of as another aspect of the industrial work routine (Lash 1984). In a consumer culture (which is conditioned by, and a reinforcement of a culture of narcissism; each being an aspect of the other) items consumed also take on a symbolic value over and above the material, economic value they are given. Consumer culture is the end product of capital accumulation whereby those concerned with profit encourage individuals through mass marketing and advertising to want more than they need. The creation of ever more needs is a necessary byproduct of the needs of capital accumulation (of which the pharmaceutical industry itself is in no way immune, as will be shown in the next chapter). This perpetuation of desire occurs precisely because the meanings of "need" and "want" have become so confounded that consumers appear no longer to be able to differentiate the two. The plethora of consumer goods circulating through our market society, the vast majority of which offer little improvement over previously existing products, has altered individual as well as social conceptions of what it means to be a self. At the same time they have 
also altered interpretations of the world outside the self. The psychic lives of the individuals that compose modern society are simply a reflection of that society itself: "man's character but mirrors the changes in a society which has not yet achieved peace with itself" (Horkheimer 1974: 27). In a society composed of little more than disposable commodities, human mental states reflect this meaninglessness as we attempt to look for meaning in the very places that negate it.

In a society where the market, based on the creation and exchange of values, mediates human relations, as well as individual conceptions of self, life becomes a form of value. As Jeremy Rifkin has pointed out, in our modern 'age of access', where the possibilities of consumption and the creation of need are endless, individuals are forced to purchase access to lived experience itself. Rifkin writes: "The distinguishing characteristic of modern capitalism is the expropriation of various facets of life into commercial relationships... The Age of Access is defined, above all else, by the increasing commodification of all human experience" (Rifkin 2000: 98 - 99). All uniquely human capabilities and desires are bought and sold on the market. In a network economy, where transactions take place almost invisibly, where the production of lifestyle is more important than actual goods, even free time and leisure are brought into the realm of the market. All human experience is commodified, and humans themselves are a market in and of themselves to be appropriated from themselves. As Rifkin points out, "the new idea in marketing is to concentrate on share of the customer rather than share of the market" (Rifkin 2000: 98). The goal of the network economy is not so much the manufacturing and selling of products as the establishment and maintenance of long term commercial relationships with consumers. In this economy, needs and wants are so 
blurred that consumers partake of commercial transactions when they may not even be aware that they are doing so since life itself is ever more becoming a commercial transaction. Culture - the shared experiences that give meaning to human life - is increasingly being commercialized and rebuilt on the need for profitable gains to be made from every aspect of human life. With a consumer culture dominating the development of the self "drugs have become a surrogate for the experience of culture itself" (Diamond 1974: 234).

It would seem that modern network capitalism has overcome the fundamental rift between capital and labour that Marx himself was so concerned about. With the proliferation of mood and personality altering drugs that effectively resocialize individuals to the reality and needs present in their lives, the capital-labour antagonism becomes a relatively minor aspect within the modern economy. In this way, psychotherapeutic drug use does not necessarily need to be seen as an intervention upon the alienating contradictions within capitalism, but a very part of the social needs of commodity production and circulation. Psychotherapeutic drug use is the product not only of standardized ways of looking at patients as expressed through the DSM and its technologies (outlined in the previous chapter), but also of the commodification of the foundation of human life itself: health.

In Horkheimer and Adorno's Dialectic of Enlightenment, which tried to explain how the principles of enlightenment rationality that espoused human freedom and emancipation eventually came to enslave human beings, they wrote: "The social work of every individual in bourgeois society is mediated through the principle of self; for one, labour will bring an increased return on capital; for others, the energy for extra labour. 
But the more the process of self preservation is effected by the bourgeois division of labour, the more it requires the self-alienation of the individuals who must model their body and soul according to the technical apparatus" (Horkheimer and Adorno 1944: 2930). Horkheimer and Adorno were hinting that the process of production within capitalism was increasingly permeating all aspects of the lives of those contained within it - and to such an extent that the self became completely permeated with its reproductive needs. Because value is itself alienated labour, and labour becomes a fundamental source of self, only through the negation of self is capitalist value created. The more and more value capitalist social relations extract from individuals, the less they become authentic human selves. This gives power not to individuals, but to capital and the individuals who possess it. Money becomes a source through which individuals try to buy back an authentic sense of self. Of course, with little success. Done on a societal-wide level, this produces a consumer society, the ultimate object of the consumerist impulse being the self we sold in exchange for our labour-power. Marx is worth quoting at length on this point:

That for which is for me through the medium of money - that for which I can pay - that am I, the possessor of money. The extent of the power of money is the extent of my power... thus what I am, and am capable of, is by no means determined by my individuality... If money is the bond binding me to human life, binding society to me, binding me and nature and man, is not money the bond of all bonds, can it not dissolve and bind all ties?... money is the alienated ability of mankind. That which I am able to do as a man, and of which therefore all my individual essential powers are incapable, I am able to do by means of money... it converts my wishes from something in the realm of imagination, translates them from their mediated, imagined or willed existence... money is the truly creative power... since money, as the existing and active concept of value, confounds and exchanges all things, it is the general confounding and compounding of all things - the world upsidedown - the confounding and compounding of all natural and human qualities. (Marx (1844) 1988: 137-140) 


\section{National Work-Life Conflict Study}

According to the 2001 National Work-Life Conflict Study funded by Health Canada, throughout the 1990s technological changes, including increased automation and the need for organizations and corporations to be "globally competitive", increased pressures on employees in ways that made it difficult, if not impossible, for individuals to fulfill multiple roles in their lives. The Work-Life study tried to account for the conflicts that occur when time and energy demands imposed by an individual's various roles (both public and private) become incompatible with one another. And while the purpose of the report was to find ways for governments, employers and employees to find balance between their work roles and their outside work life roles, its findings indicate modern minimal selfhood. Bio-political power mediates this minimal selfhood through the intervention upon the population with various coping methods, the most successful of which is psychotherapeutic drug use.

In its analysis of modern Canadian conditions of labour and life, the Work-Life study found that the demands of work have outstripped the demands of life. Further, employees donate a significant amount of unpaid labour to their employers; employees in various sectors of the economy are more likely to work unpaid than paid overtime. Whereas in 1991, 1 in 10 respondents to the Work-Life survey worked 50 or more hours a week, in 2001 this number had risen to 1 in 4 . These findings suggest that it is increasingly becoming more difficult for Canadian workers to meet work expectations during regular work hours. This modern actualization of what Marx called "surplus value extraction" has arisen, according to the authors of the study, for a number of reasons: 
Organizational anorexia (i.e. downsizing), implying that fewer employees are doing more work; a strengthening of a corporate culture which conditions people to believe that if they do not work longer hours or take their work home with them, they will not advance in their career or survive future rounds of lay offs; increased use of technology making human labour inputs superfluous, unprofitable, redundant and unneeded. For all of these reasons, in their various manifestations, role-overload - or what the authors refer to as "work-life conflict" burnout - as well as physical and mental health problems are the end result of this increasingly stressful labour situation.

The key finding of the National Work-Life conflict study was that survey respondents spent only 17 hours a week in personal, non work-related activities. Correlatively, time spent in leisure activities has decreased by close to fifty percent for both males and females with dependent care responsibilities. The drive for surplus value has extended beyond the normal working day achieved by unions, and has spread into free time itself. Surplus value, according to Marx, results from unpaid labour that is extracted from the worker by the capitalist and serves as the basis for capitalist accumulation. Surplus value is the result of the labour that workers perform for their employers beyond what is necessary to produce enough to pay for their wages. According to Marx, absolute surplus value is extracted by the lengthening of the working day, whereas relative surplus value is extracted either by lowering wages, intensifying work. However, as recent social critics have pointed out, when workers are made into consumers, the differnce in work and life becomes inconsequential since life becomes an aspect of the work routine itself. 
In all of these senses, "real" mental disorder arises from social conditions that mitigate against human self-actualization. While its expression in the form of symptoms can get classified and reclassified as was shown in chapter one, the origin of mental disorder always remains the same: the external environment. In a culture that creates fleeting desires and emotional disequilibrium, mental disorder becomes a means of individual coping. When emotional suffering cannot be actively corrected by individuals themselves through self-transformation and transcendance, psychotherapetuc drug use becomes a quick alternative to the work involved in changing one's circumstances. As the medical community increasingly perpetuates its "bio-nihilism" making individuals passive recipients of "health", increasingly individuals come to see no innate value in suffering itself, unable to glean from it any understanding of how to overcome emotionally difficult circumstances. Such is the culture of narcissism.

In the next chapter I want to understand the dynamics of the pharmaceutical industry and how it has contributed to the proliferation of psychotherapeutic drug use. Through the control of clinical trials as well as the marketing of copy-cat drugs, the pharmaceutical industry has contributed to the "creation" of illness as well as its "treatment" through chemical intervention. 


\section{Chapter Three}

\section{The Pharmaceutical Industry}

While it is true that depression and anxiety are actual psychological conditions experienced by a large number of people in North America and abroad, it is equally true that the pharmaceutical industry sells sickness itself, promotes ill health in a never ending attempt to find new categories of "sick" people - new ways of medicalizing the human condition itself. As Marcia Angell writes, "once upon a time, drug companies promoted drugs to treat diseases. Now it is often the opposite. They promote disease to fit their drugs" (Angell 2004: 86). Indeed, as Pfizer, the maker of Zoloft, states on its web site: "We at Pfizer never stop looking for innovative solutions to society's problems."

Pharmaceutical companies are actively involved in sponsoring the definition of disease and promoting it to both prescribers and consumers. As Ray Moynihan et al. write, "the social construction of illness is being replaced by the corporate construction of

disease" (Moynihan et al. 2003: 2). The pharmaceutical industry, which is even blatantly referred to as an investment industry by members within its ranks, is often compulsively concerned with medicalizing aspects of the human condition in an attempt to widen the boundaries of treatable illness in order to expand markets for those who sell and deliver medical treatments. David Healy, a psychiatrist who has extensively researched the social effects of antidepressant medications, summarizes this quite strikingly:

"... depression was all but unrecognized before the antidepressants; only about 50 to 100

people per million were thought to suffer from it. Current estimates put that figure at 100,000 to 200,000 affected per million. This is a thousand-fold increase, despite treatments supposed to cure this terrible affliction" (Healy 2003: 20). 
Within many disease categories, whether these are depressive disorders, mood disorders, or even physiologically-based disorders such as cardiovascular disorders, informal, often little recognized alliances have emerged, comprised of drug company staff, doctors and consumer groups. While often appearing to be engaged in public awareness campaigns aimed at educating society at large about the social ills of underdiagnosed and under-treated medical conditions, these alliances often tend to promote a view of their particular condition as being widespread, serious and easily treatable. Because these "disease awareness" campaigns are often linked to pharmaceutical companies' marketing strategies, they operate to expand markets for new pharmaceutical products. Alternate, often more empowering approaches emphasizing the importance of personal coping strategies are played down or completely ignored.

There is a lot of money to be made from selling people the idea that they are sick. According to IMS Health, the world's largest health data firm, the estimated total worldwide sales for prescription drugs was $\$ 400$ billion US in 2002 (IMS web site). 53\% of this $\$ 400$ billion, or around $\$ 212$ billion, was made up of prescription drugs sold to Americans (IMS web site). In Canada, drug costs are the fastest growing component of Canadian health care costs, totaling $\$ 14.5$ billion (CDN) in 2002 (CIHI 2003: 27).

These rising costs are not simply the result of rising drug prices. Increasing expenditures have been the result of the utilization of newer, more expensive drug therapies. These newer, more expensive drugs usually come in the form of drugs referred to as "me-too" drugs - drugs which are brand name variations of drugs already on the market that provide little, if any, actual improvement over older established brand products. The me-too marketing strategy is an effective way to increase profits through 
the proliferation of drugs that contribute little to the decline of illness, but that actually add to it through the creation of new illnesses. The pharmaceutical industry gets away with this through the research and development of marketing and advertising.

No longer do pharmaceutical companies make products: they make brands. According to MedAd News, the leading marketing and advertising periodical of the pharmaceutical industry, "Branding is an essential technique for reaching doctors and patients and establishing a product's value beyond its utility" (MedAd News, August 2003: 32). Patent and intellectual property laws are the weapons brand-name manufacturers use to secure market share against each other as well as the other players in the pharmaceutical industry: generic manufacturers. Generic manufacturers produce copies of brand name products once the brand products' patent expires, selling bioequivalent products at a fraction of the cost of brand products. This price competition is important for the Canadian Health Care system, which benefits from the cost savings provided by generics. Brand manufacturers do not compete on price, though they often extol the value of the free market; they compete on brand products through the use of the marketing of illness. MedAd News writes: "branding is important for creating an emotional connection with consumers. Good pharmaceutical branding creates physician and patient recognition that maximizes launch potential and helps the product withstand generic competition or survive a switch to over-the-counter status" (MedAd News, August 2003: 34)

Within the last $10-15$ years, the pharmaceutical industry has been moving away from what it calls a "research-based business model", to one that is driven more and more by the marketing of major branded products. Compelled by the need to create 
blockbuster products, or "mega-brands", and then to defend their sales against competing brand companies releasing me-too products, as well as against generic firms releasing copies of non-patented branded products, the brand-name pharmaceutical industry is increasingly using the power of branding to capture the hearts and minds of physicians and their patients.

The me-too market depends on markets that can be created, enlarged and indefinitely expanded. The larger the market is, and can be made to be, the better it is for pharmaceutical manufacturers. Me-too drug manufacturers usually target highly common, life-long disorders which need continual treatment - such as depression, arthritis and high blood pressure; the top three therapeutic classes in terms of both prescriptions dispensed and value of sales (IMS Health web site). Since it is not the business of pharmaceutical companies to cure illness and disease, but to treat their symptoms, any disorder that holds people far enough away from death, yet at the same time not close enough to medical standards of health, is the perfect market for the continued proliferation of illness and disease and the drugs manufactured to relieve their symptoms. Indeed, if there could be said to be any sort of "inner logic" to the pharmaceutical industry in its quest for profits, it would be the fact that the industry itself is a threat to health in this perpetuation of illness and disease treatment (if we follow Illich's definition of health as being simply a designation of the intensity with which individual's cope with their internal states and environmental conditions).

The movement of the boundaries of diagnostic categories, the points at which illness and wellness is clearly defined, is most noticeable in the case of hypertension and heart disease. Since the inception of the diagnosis of hypertension, it was medical 
practice that hypertension involved any measurement of blood pressure above140 over 90 (the first number, called the systolic pressure, is the upper bound pressure that blood exerts in the blood vessels when the heart beats; the second number, called the diastolic pressure, is the lower bound pressure between heart beats). But in 2003, a professional panel composed of members from the US Health and Human Services' National Heart, Lung and Blood Institute as well as the National Institutes of Health submitted guidelines changing the boundaries of what constituted "hypertension". In the Seventh Report of the Joint National Committee on Prevention, Detection, Evaluation and Treatment of High Blood Pressure, a new disorder emerged called "prehypertension", and the range of what could be measured as some form of hypertension increased to 120 over 80 and 140 over 90 . Because of this decision, thought by many to be the result of the lobbying power of the pharmaceutical industry, overnight the market for anti-hypertension drugs increased significantly. Individuals who had gone to sleep healthy, woke up in the morning to find they had a serious disorder in need of medical attention. Since high blood pressure is not so much an analysis as it is simply a description based on a reading from a medical measuring device, causes of the elevated blood pressure are not themselves included in the "diagnosis".

Employing the same method of expanding the realm of what constitutes sickness, the cut off for high cholesterol has been significantly lowered in the last 10 years. Originally, a high blood-cholesterol level was set by the medical community at anything over 280 milligrams per deciliter. A decade ago it was lowered to 240 , and today, according to the American Heart Association, anything over 200 is considered to be a high blood-cholesterol level. It is no wonder then that Lipitor and Zocor, both prescribed 
for the treatment of high cholesterol, are the top selling drugs in the world with annual worldwide sales of $\$ 6$ billion each. Outside the realm of mental illness, the expansion of illness and disease can be seen in a wide array of disorders too numerous to go into in any detail here: Hormone Therapy, Acid Reflux Disease (heart burn), Erectile Dysfunction and even Allergies are all prone to the same diagnostic elasticity of treatable disorder in need of medical intervention.

One of the cleverest ways in which the pharmaceutical industry can maintain healthy revenues is to extend the life of a blockbuster drug that is close to losing its patent protection either by making a virtually identical drug, or a new illness for an old drug, as the patent expires, and shifting users from one drug to another. The consequence of this is the perpetuation of illness and the amount of available medication needed to treat these illnesses. A quick glance on all SSRI products web sites tells the story. Prozac, for example, manufactured by Eli Lilly (the $10^{\text {th }}$ most profitable pharmaceutical manufacturer in 2003), was the first of the SSRI's initially made for the alleviation of depression. Soon after its launch, however, Eli Lilly found that Prozac could be marketed for a number of other disorders. In 1987, the FDA approved Prozac for the treatment of depression. In 1994, Prozac was approved for the treatment of obsessivecompulsive disorder; in 1996, for the treatment of bulimia; and in 1999 for the treatment of geriatric depression. And in 2003, Eli Lilly introduced Prozac Weekly, a sustained release tablet. All of this has led Eli Lilly proudly to proclaim that since its release Prozac has helped over 40 million people worldwide, making it one of the most popular antidepressant medications in history - and for the treatment of disorders not even related 
to depression. At the peak of its sales in the mid-90s, Prozac accounted for one-fourth of Eli Lilly's total revenues, with annual sales reaching $\$ 2.6$ billion worldwide.

Of course, with the unprecedented revenues of Prozac, other pharmaceutical manufacturers soon followed Eli Lilly's lead. Eli Lilly's ability to expand the possible uses of Prozac, increasing both the total number of people who appeared to have certain, specific mental disorders, as well as the number of mental disorders that could conceivably be treated with a specific drug, has been a model for other pharmaceutical manufacturers. All this further exaggerates the number of people deemed to be mentally ill as well as the total number of mental illnesses within society. GlaxoSmithKline released its own psychotherapeutic drug, Paxil in 1997; approved for the treatment of Depression, Generalized Social Anxiety Disorder, Panic Disorder, Obsessive-Compulsive Disorder and Generalized Anxiety Disorder. Pfizer, the world's most profitable pharmaceutical manufacturer, not to be outdone released Zoloft in 1999; approved for the treatment of Depression, Social Anxiety Disorder, Posttraumatic Stress Disorder, Panic Disorder, Obsessive-Compulsive Disorder and most recently Bulimia. Effexor, manufactured by Wyeth, was released in 1993, and is now approved for the treatment of Depression, Generalized Anxiety Disorder, and Social Anxiety Disorder. Finally, a newly emerging player in the pharmaceutical game, Forest Labs, even went a step further in this copycat, me-too drug development phenomenon. Forest Labs released Celexa in 1998 for the treatment of Depression, and then created a me-too of its me-too drug Lexapro, which received approval in 2002 for the treatment of Depression and Generalized Anxiety Disorder. 
Prozac lost it patent in 2001. However, this did not prevent Eli Lilly from selling Prozac's active ingredient - Fluoxetine Hydrochloride. Eli Lilly renamed Prozac Sarafem (made up of exactly the same active ingredient found in Prozac - Fluoxetine Hydrochloride) coloured it pink and lavender in an attempt to make an emotional appeal to its new users as a part of its branding strategy, and received FDA approval to market it for a brand new disorder called "Premenstrual Dysphoric Disorder." According to the Sarafem Web site, Premenstrual Dysphoric Disorder (PMDD) is a distinct medical condition characterized by the intense mood and physical symptoms that occur before a woman begins her period. Symptoms include irritability, sadness, sudden mood changes, tension, bloating and breast tenderness - all of which women presumably have been experiencing since the beginning of time. What has changed is the social context within which these symptoms are interpreted and dealt with. The Sarafem Web site goes on to state that while PMDD is not fully understood, many doctors believe it may be caused by an imbalance of serotonin - the same hypothesis that is yet to be proven as a possible cause of depression - concluding that if left untreated the symptoms can worsen with age. Further, stopping treatment may cause PMDD symptoms to return, thus creating the need for continual treatment with the drug, even if the individual's symptoms have disappeared. All this turned a very high profit for Eli Lilly: In December, 2002 Eli Lilly sold the sales and marketing rights to Sarafem to Warner Chilcott for \$295 million in cash, about 3.2 times its estimated sales for that year (Warner Chilcott Press Release December 5, 2002).

Bioethicist Carl Elliott told the Washington Post in 2001 that "the way to sell drugs is to sell psychiatric illness. If you are Paxil and you are the only manufacturer 
who has the drug for Social Anxiety Disorder, it is in your interest to broaden the category as far as possible and make the border as fuzzy as possible." These comments came in response to statements made in the journal Advertising Age by Barry Brand, Paxil's marketing director: "Every marketer's dream is to find an unidentified or unknown market and develop it. That's what we were able to do with Social Anxiety Disorder" (The Advertising Age, Vol. 3: 64). After the events of September 11, 2001, GlaxoSmithKline, playing on the seemingly all-pervasive culture of fear within the US, spent twice as much on advertising in November 2001 than they did in November of 2000 (CBC Documentary: November 5, 2003). Their just released drug Paxil quickly became one of the most popular and best selling medications in the treatment of anxiety after 9/11. According to the Fifth Estate, total advertising on all antidepressant medications in the November following the attack is estimated at around $\$ 800$ million (CBC Documentary: November 5, 2003). In a time of political powerlessness and perceived insecurity, psychotherapeutic drug use becomes an easy form of social control and a means for quelling social discontent.

So just what is this wonder drug Paxil? Paxil's active ingredient, paroxetine is a mind-altering chemical compound originally approved for the treatment of depression. It was synthetically designed in 1978 by a small Danish company called Ferrosan. In 1980, Ferrosan sold paroxetine to what would later become GlaxoSmithKline. After initial clinical trials of paroxetine seemed to indicate that the compound was less effective than older antidepressants, GlaxoSmithKline was considering discontinuing its trials and permanently shelving the product. However, in the nineties, when pharmaceutical companies discovered that more business and profit could be made 
through the marketing of disorders, rather than simply drugs, GlaxoSmithKline came up with an ingenuous marketing strategy for Paxil: The Paxil Spectrum. In the drug reference manual for physicians called the Compendium of Pharmaceuticals and Specialties (the large blue book that is present in each doctor's office - look for it next time you go) all drugs for sale in Canada are listed with their product monographs and clinical trial information. Organized alphabetically, each letter is separated by a glossy direct-to-physician advertisement. For the letter $\mathrm{P}$, there is a startling ad for Paxil. Above Greek statues of various gods, each afflicted with various modern disorders, bold faced font appeals to the physician: "Look for the PAXIL Spectrum in every patient." Under the Greek statues are the various disorders Paxil is marketed to treat: Depression, Generalized Social Anxiety Disorder, Panic Disorder, Obsessive-Compulsive disorder and Generalized Anxiety Disorder. Below the statues is the product description, written as if in bullet points: "See an anxiety disorder. Look for depression. And vice versa. You'll find up to $70 \%$ of patients could have a comorbid condition... PAXIL. No other antidepressant has more indications. Look for the PAXIL Spectrum in every patient. When you treat one, treat them all. PAXIL. See why it's the \#1 prescribed SSRI in Canada" (Canadian Pharmacists Association 2003: 1240). When GlaxoSmithKline found that the clinical trials of Paxil for the specific treatment of depression weren't going as planned, GlaxoSmithKline altered its approached to drug development and got into the business of illness development. And it worked: according to IMS Health, Paxil was the $9^{\text {th }}$ most prescribed medication in Canada in both 2002 and 2003, with close to 3.5 million prescriptions filled each year. 
But things haven't been going as planned with Paxil. Beginning at least by February of 1990, if not before (Paxil and its relatives Prozac and Zoloft have been in clinical trials and development since the mid 80's), GlaxoSmithKline and the rest of the pharmaceutical industry were aware of reports of a potential relationship between the ingestion of SSRI's and violence or suicide. The first reports, denied and initially suppressed, were usually directed at Prozac, the first SSRI approved for sale in Canada by Health Canada. However, through the work of such people as David Healy (and his unorthodox rise to fame through his much publicized dismissal - and subsequent reinstatement - from the University of Toronto-affiliated Centre for Addiction and Mental Health directorate position for making precisely the claims above) it has been recently shown that companies such as GlaxoSmithKline never fully researched or properly tested the propensity of Paxil and other SSRI's to cause suicide or violence in some people, nor did they warn the public or the medical community about these and other lesser adverse reactions to the ingestion of these drugs, such as addiction and even increased feelings of depression. How do these drugs get approved? In order to understand how so many psychotherapeutic drugs are approved for sale, one must look into the research and development of drugs and the clinical trials that are often manipulated or biased in their attempt for approval.

\section{Research and Development}

The pharmaceutical industry commonly presents itself as devoted to the research and development of new, life-saving drugs. Drug prices may be very high, but these prices are needed to fund the development of these drugs - which can reach up to $\$ 800$ million according to the industry's representative, the Pharmaceutical and Research 
Manufacturers of America (PHrMA), as well as the Canadian equivalent organization Pharmaceutical Manufacturers of Canada (Rx\&D) (The figure of $\$ 800$ million comes from a study produced by Joseph A. DiMasi entitled "The Price of Innovation: New Estimates in Drug Development Costs", published in the Journal of Health Economics (vol. 22) in 2003. DiMasi is the Director of Economic Analysis of the Tufts Center for the Study of Drug Development. The primary financial contributors to the Centre are the pharmaceutical and biopharmaceutical industries). But just what goes into the research and the development of new drugs?

Bringing a drug to market is a long process. But drug companies play nowhere as large a role in research and development as they commonly claim when trying to justify the prices of their products. Brand-name pharmaceutical companies carefully craft their involvement in research and development.

The most important and time consuming part of research and development comes from the scientific community and its attempt to try to understand the nature of the disease or illness needed to be treated. Most often, this comes in the form of analyzing what has gone wrong in the body to cause the disease or illness. This highly refined way of looking at patients and their bodies needs to be very detailed, in accordance with the epistemology of modern medicine. In the development of modern medicines, scientists usually try to understand disease and illness at the molecular level, so as to be able to intervene adequately at the believed source of the chain of events contributing to the expression of the disease through its symptoms. Most drugs, in fact, do not do anything, but rather prevent chemical or molecular episodes from occurring within the normal processes of the body. For example, SSRI's prevent the reuptake of serotonin, allowing 
serotonin - thought to be a contributer to depression, though there is still no conclusive evidence of this - to continue acting on receptors within the brain. This increases the supply of serotonin - a neurotransmitter that for reasons scientists also have yet to explain significantly alters mood, sleep patterns and pain - in the brain. By altering the action of a specific set of chemicals involved in the transfer of signals from cell to cell in the central nervous system, SSRI's allow for a sustained amount of serotonin to be available at the gaps between nerve cells, called synapses. The end result of this increased supply tends to result in improved mood - in some people. In others, it can also lead to acts of violence and suicide.

This process of learning about the disease or condition constitutes the "research" phase of research and development, often taking decades. In this time, the drug can either be made to fit current conceptions of illness, or illnesses themselves can come to fit available drugs. In each case, contrary to the claims of the pharmaceutical industry, the research of drugs to fit existing disease categories is almost always carried out at universities or government run labs in the US or Europe. The pharmaceutical industry usually steps in at the next stage: development of medicines and clinical trials.

Once the basic research has been completed - meaning more often than not that the knowledge of the disease as well as the ways of perceiving its causes and effects have sufficiently permeated the medical community to gain widespread acceptance - the search begins to discover or synthesize a molecule that will alleviate the disease. Preferably for the pharmaceutical industry, alleviation of symptoms is what is aimed for it is not in the best interests of the industry's quest for profit to cure a disease. This is the development aspect of the research and development. 
The development part of research and development is composed of two stages: Preclinical and clinical. The preclinical stage is concerned with finding promising drug candidates and the study of their properties in animals and cell cultures. Only a very small number of drug candidates that make it through preclinical development go on to be tested in humans - the clinical stage of drug development.

According to the pharmaceutical industry, only one in five thousand candidate drugs makes it to the market - one in one thousand survive preclinical testing, and of those, one in five makes it though clinical testing (Angell 2004: 23). Clinical testing is the most expensive part of drug development. The implication of this is that the vast majority of drug candidates are weeded out before the pharmaceutical industry begins devoting a substantial amount of funding into their development. So, while the pharmaceutical industry claims drug development costs upward of $\$ 800$ million, this is true for only the most expensive drugs. The actual average cost of drug development is probably closer to $\$ 150-250$ million (Angell 2004: 46), (see also consumer advocate group Public Citizen's report “Rx R\&D Myths: The Case Against the Drug Industry's 'Scare Card,'” July 2001).

The clinical stage of drug development is regulated by Health Canada, though Health Canada no longer conducts independent clinical reviews of drugs approved for sale in Canada; it now reviews the clinical trials submitted to the Health Products and Food Branch with the drug monographs provided by the pharmaceutical manufacturers. By law, before a company can sell a new drug it must prove that the drug is safe and effective. In order to do this, a series of trials is necessary. Phase I trials involve giving the drug to a small number of healthy volunteers to establish safe dosage levels and study 
the drug's metabolism and possible side effects. If the drug does well in Phase I trials meaning the presumed side effects are deemed to be relatively mild and the benefits of consumption outweigh any negative consequences - Phase II trials begin. In Phase II trials, up to a few hundred patients receive the medication for the relevant condition. The drug is given at various dosages and the effects of the drug are measured against a placebo. Interestingly enough, all drugs approved for sale in Canada by Health Canada, as well as in the US by the FDA, are approved on the basis of working better than a placebo. Instead of approving drugs through trials showing improvement over older drugs, all the pharmaceutical industry must show is that their drug works better than nothing. This has had the consequence of allowing the proliferation of drugs marketed for sale in the last 20 years. Today, it is estimated that $44 \%$ of all Americans are medicated (New York Times: November 12, 2004). Minor extensions of previously existing drugs - me too drugs - have promoted the creation and extension of pseudoillness throughout society. This will be discussed further below.

Finally, if a drug is proven to have more positive than negative characteristics for a certain population, and if there is proof that the drug works better than nothing (meaning the drug causes some effect, preferably one in line with dominant conceptions of the healthy individual, for example, causing "socially desirable" effects in depressed or anxious individuals) (Kramer 1997) - Phase III trials begin. Phase III trials evaluate the safety and effectiveness of the drug in a larger population - usually anywhere from hundreds to tens of thousands of research patients.

There is a catch to this neat schema of drug trials and development. It has to do with patents. Drug companies usually obtain a patent on a new drug before clinical 
testing begins because it is difficult to keep information about the drug secret after this point. Patents - artificial government granted monopolies in an industry that espouses the benefits of the free market each chance it gets - protect companies against competition during the clinical trial period. But because clinical trials can take anywhere from four to seven years, total patent length ( 20 years in developed market economies) is diminished. The brand-name pharmaceutical industry's need for patent protection means, in light of the fact that drug companies are investment firms obligated to their shareholders and their investments in the company, drug firms often rush these trials, contracting out drug trials and, as I will show, manipulating or even concealing drug trial data.

Drug companies often rely on contract research organization (CROs) to conduct many of their trials. In 2001, there were about 1000 CROs operating around the world, with revenues from their drug companies clients reaching approximately $\$ 7$ billion (Angell 2004: 29). These contract research organizations establish networks of physicians who, working under the organization's supervision, are paid to administer the drugs under study and to collect all relevant information on their effects. However, this perceived transparency is not always the case. The leading provider of diagnostic testing and information is a publicly traded corporation called Quest Diagnostics. The bulk of its clinical trials (about one-third) are done for GlaxoSmithKline, the makers of Paxil. GlaxoSmithKline holds approximately 20.9 million shares, or $23 \%$ of the outstanding shares of Quest Diagnostics. As part of the transaction, GlazoSmithKline has the right to designate two members on the Quest Diagnostics Board of Directors and has agreed to certain standstill provisions in connection with its ownership of Quest Diagnostics 
common stock (Quest Diagnostics web site). According to Forbes.com, Quest Diagnostics had made $\$ 440$ million in profits in 2003 .

The number of clinical trials conducted in the US in 2001 was estimated at 80,000, with 2.3 million Americans serving as human subjects (Angell 2004: 222). In Canada, over 100,000 Canadians volunteered in drug trials in 2003 (Ottawa Citizen, July 13, 2004). Three years ago, there were 600 new drug trial applications in Canada; in 2003 that number had doubled to over 1200 applications (Ottawa Citizen, July 13, 2004). This solicitation of highly needed patient volunteers also contributes to the proliferation of drugs throughout society. Potential subjects are solicited in various ways - postings in health-related Internet sites, television, radio and newspaper ads, bus stop posters etc. This is usually done though a public relations group to make the industry's presence less noticeable or clear. Solicitations are also often made as public service announcements, purporting to be educational campaigns about an illness and the need for a cure to which the clinical trial underway is contributing. Perhaps the most clever means of perpetuating illness and needed therapies for its mediation occur by drug companies setting up patient advocacy groups as magnets for people with specific illnesses and disease. The creation of these groups provides a large pool of possible drug trial candidates as well as allowing for the spread of discourses about the associated disease or illness. According to Marcia Angell, most human subjects are recruited through these means, not referred by their doctors. Compensation for participation in clinical trials ranges from a few hundred to a few thousand dollars. 


\section{Patient Advocacy}

Because direct-to-consumer advertising is heavily regulated within Canada (though direct-to-consumer advertising budgets in the US have nearly tripled between 1997 and 2001 - the percentage of total advertising accounted for by television increasing from 25 to 64 percent - and this advertising flows across the border through easy Canadian access to US magazines and television stations (Angell 2004: 234)), the pharmaceutical industry has found a clever way to advertise its brands in Canada: Patient Advocacy Groups. According to Canadian federal law, when advertising in print and electronic media, the pharmaceutical industry can only advertise the name of a product or say what a product treats, but it cannot do both at the same time. So, according to Alan Cassells, a Canadian health policy researcher, "the best way to advertise is to make your promotion not look like advertising... and one of the ways you get your promotion to not look like advertising is to use... consumer groups to speak for you" (Toronto Star, August 12, 2004). At a recent pharmaceutical conference in Toronto, a public relations expert made a presentation concluding that sales rise when drug companies develop partnerships with patient groups.

These groups become a public face for disorders, often espoused as places where stigma can be overcome and knowledge about disease and illness attained. Often, however, drug companies fund studies, use psychiatrists to affirm a new disorder as a farreaching problem and fund these advocacy groups to place pressure on provincial governments to cover needed drugs on their drug benefit formularies. An example of this sort of technique is present in the advocacy group CHADD - Children and Adults with 
Attention Deficit/Hyperactivity Disorder, an organization that was founded in the US in 1987, and subsequently expanded to Canada in 1991.

CHADD is a patient support group which allows parents and their children to access information on Attention Deficient Disorder (ADD) and Attention Deficit and Hyperactivity Disorder (ADHD). CHADD has been an integral group in the perpetuation of the belief that ADD and ADHD are real illnesses that afflict a large number of children $-10-20 \%$ of all children in the US alone, equaling over 8 million children afflicted - that can be successfully treated through medical intervention. Indeed, ADHD is now thought to be the most studied childhood psychiatric disorder. CHADD has been at the forefront of this understanding. But this public service isn't all that it appears. Since 1988, CHADD has received over one million dollars in funding by a brand name pharmaceutical company named Novartis. Novartis' leading product: Ritalin. In the mid-nineties, CHADD lobbied Washington to reclassify Ritalin as a Schedule III drug - a drug that is relatively benign and has no addictive qualities - from a Schedule II drug - a drug that is considered potentially addictive with strict production quotas on its distribution. The Drug Enforcement Agency eventually ruled against the proposal, but not before directly implicating Novartis in having to gain from the widespread distribution of the drug had Ritalin been rescheduled. In a Ritalin background paper, the DEA wrote: "The DEA has concerns that the depth of the financial relationship with the manufacturer [Novartis] was not well known to the public, including CHADD members, that have relied upon CHADD for guidance as it pertains to the diagnosis and treatment of their children." 
In the 1950s, doctors and psychiatrists working in the framework constructed by a researcher named of George Still - a popularizer of the study of childhood disorders as primarily biologically-based - diagnosed restless and inattentive behavior in children as the result of "minimal brain damage". In 1980, the American Psychiatric Association relabeled this disorder Attention Deficit Disorder (with or without Hyperactivity), and then again in 1987 (the same year CHADD was formed) as Attention Deficit/Hyperactivity Disorder. This most recent name change places emphasis on the importance of hyperactivity as one of the central features of the disorder in addition to inattention and impulsiveness. In line with the diagnostic criteria laid out by the DSM, doctors and parents have come to believe that the central deficiency in ADHD children is an inability to regulate their own behavior. As with the scientific leanings of the DSM, all social and environmental causes of the disorder are ignored. The Diagnostic and Statistical Manual of Mental Disorder, Fourth Edition, has cumulated all the various behaviors of children with ADHD and listed 9 trademark symptoms of ADHD: 1. Often has difficulty waiting their turn; 2. Interrupts or intrudes on others; 3 . Blurts out answers before questions have been completed; 4. Has difficulty playing or engaging in leisurely activities quietly; 5. Cannot remain seated for small to moderate periods of time; 6 . Runs or climbs about at times; 7. Fidgets or squirms; 8. Talks excessively; 9. Is often on the go. These DSM criteria of ADD and ADHD are symptoms of childhood itself, not a brain disorder. ADD and ADHD are diagnoses of a society unable to raise children without the manipulation and alteration of universal characteristics of childhood through behavior modifying drugs. Parents are too busy (or perhaps too depressed, anxious and afraid of social situations) to raise their children in ways that would give them the socially 
desirable characteristics the DSM would imply: Passivity, lack of creativity, restraint and self-control - not coincidentally, all characteristics deemed necessary for adulthood and the world of employed labour. Even the US National Institute of Mental Health writes on its Web site: "There is little compelling evidence at this time that ADHD can arise purely from social factors or child-rearing methods. Most substantiated causes appear to fall in the realm of neurobiology and genetics."

There is a wide array of governmental approaches to the monitoring of ADD and ADHD, most of which appear on consumer advocate and pharmaceutical company "educational" Web sites. Some of the more popular rating scales used in the assessment of these "disorders" are: The Conners Teacher Rating Scale (CTRS) and the Conners Parent Rating Scale (CPRS), The Comprehensive Teacher Rating Scale (ACTeRS), The ADHD Rating Scale, The Child Attention Profile, The Child Behavior Checklist (CBCL), The Home Situations Questionnaire, The School Situations Questionnaire, and The Academic Performance Rating Scale (APRS). As with the Hamilton Rating Scale for depression, one of the most popular methods of diagnosis of ADHD is a flimsy, subjective scale with a large gray area to fit children into various categories. Through the use of the Attention Deficit Disorder Behavior Rating Scale (ADDBR) observers rate children's behavior (i.e. the presence of fidgeting) on the basis of "not at all", "just a little", "pretty much", or "very much" criteria. The results are then compared with US norms to indicate whether or not a disorder exists in the child. Obviously, the ADDBR lacks rigidity. There are no set cutoffs between each level, and therefore much is left open to the observer's discretion and personal bias. What is considered "pretty much" to one observer may only be "just a little" to another. This may place many children in a 
gray area - allowing for common childhood activities such as the deplorable "climbing around" symptom found in the DSM, to be medicalized and intervened upon with medicine.

Another consumer advocate group, the Anxiety Disorders Association of America (ADAA) was founded in 1980 as the Phobia Society of America, and changed its name to ADAA in 1990 to encompass a full range of anxiety disorders - Generalized Anxiety Disorder, Social Phobia Disorder, Post-Traumatic Stress Disorder and Obsessive Compulsive Disorder, amongst others. Again the main contributor to the advertising and "education" campaigns of this group is GlaxoSmithKline, the makers of Paxil.

\section{Doctors and Conflict of Interests}

Another aspect of illness creation and perpetuation includes doctors. Doctors are often paid large sums of money by the pharmaceutical industry to enroll patients in clinical trials. The average amount spent in 2001 for this type of recruitment was $\$ 7,000$ per patient. It is obvious that this sort of practice allows for the possibility that doctors may enroll "borderline" patients into studies when, in fact, they do not fully fit the diagnostic criteria of the disease or illness completely (assuming that the disease itself is not in large part the creation of medical perception). This has two consequences: the diagnostic criteria of disease and illness expand to include patients who previously did not fit these criteria; and patients who aren't necessarily ill are being used to test and approve drugs for sale in the US and Canada.

To decide which drugs to prescribe for their patients, doctors often rely on sources of information claimed to be objective and unbiased, including both drug company marketing and the drug conferences pharmaceutical manufacturers organize. But the 
most significant source of information for doctors comes in the form of medical journals which present new scientific advances and their interpretation. Medical journals are the most valuable guide allowing access to newly released clinical trial results. But often these publications are themselves biased in ways favourable to pharmaceutical manufacturers, mainly because drug companies have a considerable amount of control over the way research is carried out and reported. Whereas in the 1980 s researchers were largely independent of the companies that sponsored their work, which allowed them an academic freedom and integrity, now companies are involved in just about every detail of the research - from the study design through to the analysis of the data, to the decision on whether or not to publish the results (something the pharmaceutical industry is not required by law to do). Academic centers are now partners with the pharmaceutical industry.

For example, Apotex, the largest generic manufacturer in Canada, gave the University of Toronto's Faculty of Pharmacy $\$ 5$ million dollars so it could establish two new educational facilities designed to "produce Canada's next leaders in pharmacy research and professional practice" (U of T Faculty of Pharmacy web site). The donation led to the creation of the Apotex Resource Centre and the Apotex Multi-Media Classroom (and perhaps a few more prescriptions filled with Apotex product by graduating pharmacists). Prior to this donation, Apotex gave a total of more than $\$ 1.4$ million in support of student financial aid and educational initiatives, such as the University of Toronto's Doctor of Pharmacy program. GlaxoSmithKline has also donated $\$ 1$ million to the University of Toronto's Faculty of Pharmacy to establish a Chair in Pharmaceutics and Drug Delivery - a very academic way of saying 
GlaxoSmithKline has purchased the right to access clinical trials and drug development through the University of Toronto. According to Paul Lucas, GlaxoSmithKline's president and chief executive officer, this donation was part of GlaxoSmithKline's $\$ 10$ million dollar Pathfinders Fund to create research positions in all 16 Canadian medical schools as well as in other university faculties across Canada.

The implication of these generous contributions to universities is the expropriation of academic freedom in the name of pharmaceutical interests.

While working at the University of Toronto-affiliated Hospital for Sick Children (HSC), Dr. Nancy Olivieri signed a contract, which included a confidentiality clause, to test a new drug for the Apotex pharmaceutical company. Upon discovering that some of her child subjects were experiencing high levels of iron overload that could compromise liver function and lead to life threatening liver cirrhosis, Olivieri immediately stopped the tests and insisted that these health risks be communicated to her patients' parents. Apotex not only refused to do this, but also halted all further drug trials at the Hospital for Sick Children, confiscated the trial medicine, fired Olivieri from the study, and threatened her with legal action if she divulged any information to her patients or their parents.

Acting on her ethical obligations and confident that the University of Toronto and the Hospital for Sick Children would support her, Olivieri informed her patients of the risks. The events that ensued would later be referred to by the Globe and Mail as Canada's worst academic and research scandal in decades. Olivieri and her few supporters began receiving anonymous threatening letters that were later proven to have been sent by co-worker Gideon Koren, professor of pediatrics, pharmacology, pharmacy, 
medicine, and medical genetics at the University of Toronto and a recipient of Apotex funding. Koren also sent anonymous letters containing unfounded allegations against Olivieri to the media and the Hospital for Sick Children disciplinary committee. Apotex and certain senior hospital and University employees later used these allegations as a basis to level misconduct charges against Olivieri. Apotex also used these allegations in attempts to discredit Olivieri and to argue that claims against their drug were therefore unfounded. Disturbingly, Olivieri's employers initially kept the existence of these allegations secret from her, providing her with no opportunity to defend herself or to clear her reputation.

Six years after the first signs of problems with the Apotex drug were detected, an Independent Committee of Inquiry completely exonerated Olivieri from all allegations of misconduct. The committee's extensive report, (over 500 pages with over 1400 footnotes referencing the extensive paper trail from the incident) arrived at 31 recommendations, including the banning of secrecy clauses in research contracts. The report was critical of the University of Toronto and the Hospital for Sick Children for their failure to protect Olivieri's academic freedom. At the time Olivieri came under attack, the University was in negotiations with Apotex for a $\$ 20$ million dollar infrastructure investment.

Another example of this sort of practice occurred when Dr. David Healy, a wellknown scholar at the University of Wales College of Medicine who studies SSRI's and their effects on different patient groups, made comments on the suppression of clinical trial data regarding the effects of SSRI's on rates of suicide in those prescribed them. Healy communicates his story in his latest book, Let them Eat Prozac (Healy 2003). In September 2000, Healy accepted a job offer from the University of Toronto Centre for 
Addiction and Mental Health (CAMH), which had been actively trying to recruit him since July 1999. Soon afterwards, he appeared in Toronto as one of several distinguished lecturers to an international colloquium on the history and future of psychiatry. Healy's talk addressed concerns about conflicts between accepted clinical practice in drug testing and growing private influence in public research. He likened large pharmaceutical companies to tobacco companies, in that they may be suppressing research that could demonstrate hazards resulting from the use of their products.

Healy's research, using data from Prozac manufacturer Eli Lilly, led to estimates that as many as 50,000 people have committed suicide on Prozac since its launch, over and above the number who would have done so if left untreated. The suicides were believed to be due to a side effect known as akathisia, an inner restlessness that can lead to violent and suicidal behaviour in some individuals on SSRI's. Following this lecture, which received the highest participant evaluation of all presenters and panelists at the colloquium, Healy was contacted by the University of Toronto and told that his job offer had been withdrawn. Faculty organizations such as the Canadian Association of University Teachers and the University of Toronto Faculty Association believe that Eli Lilly's major financial contributions to the CAMH were behind the Centre's decision. Ultimately, Healy was completely vindicated when the University and the Centre agreed to a settle a lawsuit that he launched against them. Further, in clinical trial data released in the spring of 2004 by major SSRI manufacturers, it was indeed found that suicide does increase in certain populations using these drugs - something clinical data provided by the pharmaceutical companies concerned proved. All SSRI medications in the US, 
Britain and Canada now have black box warnings of an increased risk of suicidal thoughts and behaviour in children and adolescents.

Having to compete for funding with CROs, universities now accommodate themselves to the pharmaceutical industry by letting pharmaceutical companies control the way clinical trials are carried out (Healy 2003). Sponsoring companies keep all data of clinical trials and may not even let researchers themselves see all of it once the trials are complete. They also analyze and interpret the results, and decide what, if anything, should be published in the very medical journals doctors rely on for prescribing information and advice. Authors of a survey of the academic policies surrounding pharmaceutical industry clinical trials and academic institutions write: "Academic institutions rarely ensure that their investigators have full participation in the design of their trials, unimpeded access to trial data and the right to publish their findings" (Quoted in Angell 2004: 103). In the game of pharmaceuticals, rarely are researchers the objective, scientific representatives of the advancement of medical knowledge they often claim to be. Instead, at least in the development stage of the research and development of a new drug product, they should be seen as consultants to the pharmaceutical industry's drive for quick drug development. If a clinical trial or research study needs a big name on its title page, trials and studies can be sent to high profile physicians for comments and this will qualify as authorship. This phenomenon is called "ghostwriting". According to David Healy, "at least 50\% of academic publications in therapeutics are now ghost written" (Healy 2003: 14). If this hurts the medical community's conscience it definitely doesn't hurt its bank accounts: the head of the department of Psychiatry at Brown university medical school made over $\$ 500,000$ in consulting fees in 1998 . Further, 
according to David Healy, independent research in psychopharmacology stopped around 1980 and, if there had been independent research conducted, drugs such as Prozac would never have become the phenomenon it turned out to be (Healy 2003: 14).

Bias in clinical trials can come in a number of different forms - all of which permeate the very medical journals doctors need to gain the latest information in drug development and prescribing. Often, bias is a matter of the marketing spin manufacturers choose to incorporate in clinical trials themselves. Researchers, both during the campaign to enroll clinical trial subjects as well as after drug development and approval, can hype a drug that does not live up to the support they give it. Or bias can be built into the study design itself - something that is evident in all placebo-controlled clinical trials which allow all new drugs to appear effective, when in fact they could be less effective than older types of products already on the market. Obviously, biased and inaccurate clinical trial data is of little use to doctors who need to know if a newly released drug is an improvement over what they are already prescribing. This ever growing number of drug products on the market for therapeutic use has the consequence of setting up a situation where there could conceivably be one drug for each person without any undesirable side effects. If one drug does not work as effectively as is deemed medically necessary, there will be countless more ready and waiting to be prescribed so that the therapeutic effects occur precisely as they are meant to. With regard to psychotherapeutic drugs which can significantly alter an individual's mood, personality and self-perception, the consequences of this phenomenon are potentially politically and socially catastrophic. 
Further, trials can be designed to be too brief to be meaningful in real world application. Antidepressant trials are often conducted for only a few weeks, yet patients in the real world may take these kinds of drugs for many years. Some treatments may look positive on their own over a short period of time, but may not be necessarily effective over the long term. Indeed, psychotherapeutic drugs may even cause disorders. David Healy writes: "There is plenty of evidence that antidepressants can be shown to do something in the short term but almost no evidence that things turn out better in the long run, and there are many reasons to believe that we [the medical community] may be making things worse." (Healy 2003: 43) Or, perhaps the most common way to bias trials is to present only part of the data, publishing the first few years of the trials which may make the new product appear to be beneficial, and withholding the remaining results of the study, or as in the case with Nancy Oliveri, completely suppressing negative results. As a case in point, GlaxoSmithKline was found guilty of withholding clinical trial data on Paxil. A confidential internal document published in the Canadian Medical Association Journal acknowledged that clinical trial results were "insufficiently robust" to warrant an application for use in depressive patients under the age of 18 , and went on to suggest that the company "effectively managed the dissemination of this data in order to minimize any potential negative commercial impact." The memo also stated that only positive findings would be reported from a clinical trial conducted by a company in the US that found Paxil was no more effective than a placebo: "It would be commercially unacceptable to include a statement that efficacy had not been demonstrated, as this would undermine the profile of Paxil." (The Toronto Star, February 13, 2004: A20) 
The case of antidepressant medication is telling. In a study published in 2002, Psychology professors Irving Kirsch and Thomas J. Moore, using the Freedom of Information Act, obtained FDA reviews of every placebo-controlled clinical trial submitted for approval of the six most widely used antidepressant drugs between 1987 and 1999 - Prozac, Paxil, Zoloft, Celexa, Serzone and Effexor. These drugs totaled $\$ 8.3$ billion in worldwide sales in 2002 . Their findings are disturbing: Of the 47 trials conducted for the six drugs, only 20 showed any measurable advantage over placebos. Typically, most of these trials lasted for just six weeks. But Kirsch and Moore's main finding is even more startling: On average, placebos were $80 \%$ as effective as the top selling antidepressants. Further, the difference between the drug and placebo was only about two points on the 62-point Hamilton Depression Scale, which measures the severity of depression. Clinical trials are a game for drug companies to win, rather than a venue for generating scientific knowledge. At a cost of perhaps a million dollars a day, not getting approval for sale is unacceptable in an industry dominated by the pursuit of profit.

Why this high placebo response? According to Donald Klein, the well-known psychiatry professor at Columbia University's New York State Psychiatric Institute, “the problem with antidepressant studies is that anything that can be confused with ordinary unhappiness gets in" (Greenfield 2003: 81). This means that subjects in clinical trials are insufficiently depressed or too close to "normal" to show dramatic improvement. Relatively socially adjusted, energetic yet moderately depressed people who are more likely to finish a six week trial are more likely to register a high placebo response. Since there are no objective biochemical markers or blood tests for the presence of depression 
or its degree, its presence is left up to the authority of clinical practitioners and the Hamilton Rating Scale for Depression. So, if the clinician rates the presence of depression in a client liberally, more people will satisfy the entry criteria of these trials (set at 17 points - the line dividing mildly and moderately depressed patients).

Another intriguing answer is given by Irving Kirsch. Kirsch thinks that perhaps the widespread marketing and advertising about the therapeutic salvation offered by psychotherapeutic drugs may in fact shape people's expectations, leading them to "cure" their illness in ways their medications are stated to. As Kirsch says: "The hopelessness of depression is the expectancy that a terrible state of affairs is not going to get better. Now you give somebody a treatment that's been touted as the cure for the worst things in their lives. What that does is to instill hope, which is the opposite of depression" (Kirsch 1994: 12). The implication of Kirsch's work is profound. While drug companies may have marketed their psychotherapeutic drugs beyond what the statistics given to the FDA would seem to justify, the barrage of marketing and advertising undertaken by pharmaceutical manufacturers may have inadvertently amplified the placebo affect throughout society, thus increasing the effectiveness of the drugs they promote.

In 2002, the combined profits for the top ten drug companies in the Fortune 500 (totaling $\$ 35.9$ billion) were more than the profits for all other 490 businesses put together (totaling $\$ 33.7$ billion). The median profit rate for all industries as a percentage of total sales was $4.6 \%$ for 2003 . The profit rate for the pharmaceutical firms in the Fortune 500 was $14.3 \%$. For the top ten companies, research and development amounted to only $14 \%$ of sales in 2000 . However, marketing and administration made up $36 \%$ of sales - two and a half times the expenditures on research and development (Angell 2004: 
211). Pharmaceutical manufacturers are marketing machines. Indeed, if the provisional thesis above claiming that the placebo effect present throughout society is caused by marketing and advertising has any truth to it, marketing and advertising itself should be considered an integral aspect of drug development. In their attempt to "inform and educate" patients through advertising campaigns about the disorders they may be suffering as well as the possible treatments for them, the pharmaceutical industry is expanding the penumbra of disorders into the human condition itself, and correlatively trying to intervene and correct it in socially and medically acceptable ways. Whatever the truth about placebos, individuals are increasingly being told common aspects of being human are forms of illness needing treatment. Whether the intervention takes the form of psychiatry, psychotherapeutic drugs or placebos, individuals are being reconstituted in ways unimaginable 20 years ago. The next chapter will attempt to elucidate philosophically what this reconstitution means for the self in society. 


\section{Chapter Four}

\section{Philosophy of Self and Being}

The previous chapters have explored the reasons why psychotherapeutic drug use has become so prevalent in today's society. This chapter will analyze, in a rather philosophic way, the consequences of such a practice. The argument presented is that through the increase in psychotherapeutic technologies of the self, mood (being the fundamental determinant of human understanding) is being reconfigured at the expense of understanding, furthering technological nihilism.

"What does it mean to exist at all?" This is the single question toward which Heidegger devotes his work Being and Time, published in 1927. Heidegger's question is not whether something does or does not exist, or how to catalogue the characteristics of particular kinds of things that exist. Heidegger is trying to comprehend something more fundamental, or in Heideggerian terms, something more primordial; he is asking about the very meaning of Being itself, something which I believe the widespread use of psychotherapeutic drugs again calls into question. However, nearly 500 pages later, and with only about a third of his discussion completed, Heidegger abandons this question, claiming he had been too immersed in the language of metaphysics, placing too much emphasis on "man", and not enough on Being and its historicity. Perhaps it isn't coincidental that shortly after making this decision, Heidegger joined the Nazi Party. However, I do not plan on cascading into the depths of Heideggerian philosophy but plan to use Heidegger as a starting point for dealing with questions regarding "selves". The analysis of Being found in Being and Time, as well as Heidegger's ruminations about technologies or "techne", will be the template for an understanding of the remaking and 
reconstitution of the modern self in light of the proliferation of the techne attributable to psychotherapeutic drugs.

For Heidegger, techne is not a way of "making something" through the use of technologies available to us through the application of certain technologies, but rather as a fundamental way of "revealing truth"; a way of knowing. I believe the spread of psychotherapeutic drugs reveals assumptions embedded in modern understandings of what it means to be human and in so doing opens them up to alteration and manipulation. This leads me to Foucault's concept of "Technologies of the Self" - the specific practices by which subjects constitute themselves within and through systems of power - which I believe is heavily dependent on Heideggarian ideas.

Heidegger's project in Being and Time is to lay out, in phenomenological terms, $a$ priori existentials that allow for an understanding of what it means to be as a human being. Traditional ontology, according to Heidegger, had grounded Being in causally self-sufficient source (i.e. the mind-body dualism of Descartes), yet all beings are only intelligible in terms of the structure if one kind of being - that intelligibility characteristic of the type of being that can reflect upon its existence in the first place: Human Beings. In order to discuss this further, Heidegger employs the term "Dasein", the combination of "there" and "to be" to refer to a type of being represented by human existence that, in its social and culturally bound activity, is innately self-interpreting. Each Dasein must understand itself within some culture that has already decided on specific possible ways of being human, what human capabilities and limitations are. The most that a Dasein can do is "raise to its consciousness", or clarify this interpretation in the culture. For this reason, Heidegger will conclude that Dasein has no nature, but is constantly self- 
interpreting within the bounds laid down by its culture - thus the famous maxim: "The essence of being lies in its existence."

For Heidegger, asking the question "what does it mean to exist at all?" is itself a means toward overcoming nihilism - nihilism being the denial that human existence is meaningful. Not asking this question, or some less "philosophical" variant such as "why do I exist?" emphasizing personal existence, either implies meaninglessness is the only "meaning" comprehensible to human existence or that individuals themselves posit their own absolute meaning in the world. However, it is Heidegger's contention that humans are "world disclosers", meaning that by means of our ability to use such things as tools and language as well as through our coordinating practices, we open up coherent, distinct contexts or worlds in which we perceive, act and think (Dreyfus 1991). Heidegger's analysis of Being begins with the argument that Being itself matters, and Heidegger carries out this project by analyzing the meaning attributable to being rather than simply by enquiring into whether or not a being is knowable or whether or not access to this knowledge matters at all. Indeed, Heidegger claims that simply because the question of meaning is itself capable of being asked and responded to in the first place makes the meaning of Being necessarily "real." Further, the occasional feeling of meaninglessness that each person no doubt has experienced even for brief periods in life is proof itself that one is and can question what it means to be at all, regardless of one's interest in philosophy.

Heidegger goes on to ask: "Why are there beings at all instead of nothing?" Heidegger's answer, of course, is that the concept of nothing (and thus its problem) only comes into existence with human beings. Nothingness is a creation of Being, of human 
existence. "Anxiety reveals the nothing," according to Heidegger. The realization that we could just as likely not exist, that death is the fundamental unifying occurrence that binds all peoples together regardless of culture or place, discloses to us the dreadful reality of the transience of life. The realization of this fact is termed "authentic existence." To deny the mortality and transience of one's life is to live in the illusion of inauthentic existence: existence covered up or concealed and only seen by the inauthentic individual as how others perceive it.

Heidegger does not view the world in which we live as being somehow removed from, or as being over and above, or underneath human existence. Indeed, Heidegger's terminology "being-in-the-world" implies that being and the world necessitate each other. The concepts "world", "self", "fear", and "understating" are not somehow objective entities removed from the type of subjective concern necessitated by them. Rather, each is a way in which one's existence expresses itself, a way in which we are made aware of the world of which we are a part and through which we are constituted. For human beings, our self does not end at the horizon of our skin; it extends beyond into the world of which we are a part and from which we receive direction. Heidegger calls these modes of existence "existentials". An analysis of existentials reveals what it means to Be.

Heidegger's search for existential analytics leads him to posit two ways of Being that are necessary for any awareness of self: State of mind, or mood (attunement); and verstehen, or understanding.

Existentials are not the result of abstraction from experience; they are presupposed in experience and are what make experience itself possible. Further, 
Heidegger's existential analytics are an analysis of the a priori conditions under which one's existence is made meaningful in the first place. In its totality, this is what Heidegger refers to as "fundamental ontology."

Important to the Heideggerian system is the differentiation between ontological enquiry and ontic inquiry. The first, the ontological, attempts to study being and its meanings as revealed to existents within the world; the other, ontic enquiry, instead studies entities through the use of categories - the distinguishing characteristic of the scientific outlook, and as has been discussed, diagnostic psychiatry as expressed in the DSM. However, according to Heidegger, science presupposes an understanding of being itself. It cannot be used to study being when it is Being itself that gives science its basis. Here it can be revealed how the diagnostic system itself is an articulation of a deeper attitude toward Beings in general found within the scientific and medical communities one that posts life as humanly meaningless in its attempt to catalogue and classify all reality in terms of laws and forces beyond human control.

Science is itself a way of Being in which Dasein directs its attention towards the world in a scientific way. The problem is that humans themselves cannot be seen in an ontic way without removing the very ground upon which humans are human: Selfinterpretation. Heidegger claims that science views the world as "present-at-hand"; that is, things in the world are seen as independent of their function, or usefulness to human life. This relationship is derived from experience, not fundamental to experience. When this kind of understanding loops back on human existence and becomes the ground upon which life itself is viewed, the question of what it means to Be is necessarily forgotten. Scientific, ontic understanding removes and inhibits the very ground of what it means to 
be human. Correlatively, existence necessarily becomes inauthentic and groundless because the human element within this outlook negates it: Thus life becomes nihilistic.

Heidegger claims that any science always presupposes an understanding of Being. Science cannot understand what it means to exist as a human being: It can only infer generalizations from the practices of daily life which in part apply to the ways in which humans live and understand the experiential elements that come to form who they are as individuals. For Heidegger, the primordial, or ground of all philosophical endeavor, is not in the asking of what a being is, or how to understand it through its classification or cataloging, but what it means to be at all - how the world opens up and reveals itself to Being. Asking about what technologies of the self people are either persuaded to resort to in a time of "psychic crisis", or consciously pursue in order to live "anxiety free" within society is itself a pathway into understanding this opening up of the world to human Beings. The dominance of the scientific and medical outlook says something about what sort of truth human beings are living by.

Scientifically, the fact that we are on earth, or that objects will fall due to gravity is not an a priori type of knowledge, but is learned through experience. Humans have conceivably always known that objects will fall when they are dropped; what science does is remove the human subject and universalize this as a law. Indeed, the consequences of things dropping are no longer an issue; only the measurable speed at which certain objects drop is etc. Gravity as a universal law of science does not care what is dropped, its value or cultural significance; it only cares to describe the measurable characteristics of such an occurrence. Science catalogues and divides the world and its objects according to genuses, classes and species, all of which does not 
include our human connection with these things. However, for Dasein the "in" of the term being-in-the-world refers to an ability to have things we can relate to, care about, which contributes to our sense of belonging. Being-in-the-world suggests that we are innately meaning positing and we make the world familiar to ourselves through this relationship: We make the world our home. Nihilism, as the "uncanniest of all guests" means we become strangers in our own house. What began as a means for understanding the world in which we live has become an alienating source of confusion and meaninglessness. The ground upon which meaning is constructed has become inaccessible to human beings.

This innately nihilistic scientific understanding of something primordial and ontological has the consequence of removing individuals from the world in which they live, experience, cope and feel. With respect to psychotherapeutic drug use, I argue that one existential analytic - mood - is being favoured over the other - understanding. Mood provides the background for the basis of the specific events that can affect us (Dreyfus 1991: 174). Mood discloses something in the world as mattering to Dasein in a particular way. This in turn opens Dasein up to the world, becoming a lens through which the world is perceived and acted within. In this way, mood should not be thought of as simply an "inner condition" found within individuals, but as a way of being itself a way of relatedness to the world we are in. Rather than understanding as a means for orienting oneself in the world, mood is now favoured as a way of direction. Hence the need for mood-altering drugs within modern society.

But for Heidegger, mood never reveals the full meaning of our existence. Mood ultimately shows to human existence its submissiveness in the world, what Heidegger 
will call our "throwness". We make sense of our existence as being-in-the-world by recognizing the influence of our own projecting (the ability to follow through with the possibilities of existence within us) as well as the "giveness" of our fate which we can influence through understanding. With the proliferation of dependence on psychotherapeutic drugs, this understanding is not taking place. Our fate is decided for us before hand. Mood, as the lens through which we view "things" in the world is being retuned by the medical community, resocializing those on psychotherapeutic drugs to view the world in a more "endurable" manner. However, the world becomes coloured with false pastels and no longer are individuals in command of the creativity of the art work that was once their life, but are mere recipients of tragic and pre-arranged canvases. In short, mood is a way of finding oneself in the world, but mood cannot tell us what it has found, nor explain it to us. Mood "tunes" understanding. Without understanding, this attunement is controlled and set by forces beyond individual autonomous control. Further, what those on psychotherapeutic drugs are "tuned into" is set by the dictates of the needs of the social structure - whatever this may be, whether consumerism or totalitarianism. Whatever the attunement, there can be no such thing as a free-thinking individual on psychotherapeutic drugs.

By understanding, Heidegger does not mean textbook knowledge about something, but uses the term to denote know-how within the world; understanding is the ability to do things, to seek out possibilities, not simply to know about possible things that could be done or undertaken. Understanding implies involvement within the world. The consequence of the decline in importance of this analytic of existence is that psychotherapeutic drugs themselves add to the nihilism from which they came, in that 
they remove individuals from the world of coping and from their ability to situate themselves actively in their world and to manipulate it through their own work - the basis upon which understanding originates. Psychotherapeutic drugs are not empowering as is often claimed since they remove individuals from their "thrownness" - the need for Dasein to take a stand on itself as it finds itself within the world; in short, to become an individual. If mood reveals possibilities for human action, understanding allows for individuals to take a stand on them and to pursue possibilities as individuals deem appropriate. With psychotherapeutic drugs, understanding is inserted, through the resituating of mood, by the medical community. Possibilities for action of individuals are decided before hand for them. As Hacking suggested, the labeled come to act out their label.

Peter Kramer, psychiatrist and author of the widely popular book Listening to Prozac, suggested that psychotherapeutic drug use can be considered an attempt by individuals to reshape and alter their personalities through what he calls "cosmetic pharmacology." In this way, mood altering drug use can be thought of as simply a product of social pressures to conform with existing personality traits deemed to be socially acceptable. However, Kramer does not see how the conditions in which these so-called choices are made are conditioned for individuals beforehand, nor does he bother even to approach the issue. In Kramer's book, a number of examples are pursued to elucidate the fact that the idea of the self is being reconstituted by the medical community through psychotherapeutic drugs. In one example, a patient named Julia is being treated with psychotherapy, which seems to have improved her depression and inability to handle her levels of stress at home and at work, though has not completely "cured" her of 
them. Julia has been seeing a psychiatrist for close to a year for these issues. After the release of Prozac, the first of the blockbuster SSRI's, Julia is treated with it for five months and, as if by divine intervention, is able to cope with her stress and depression she begins to excel at work and her marriage reasserts itself when it had been on the verge of collapse. Soon after, Julia withdraws from counseling and becomes dependent solely on her medication as a source of foundational strength in her life. On Prozac, Julia claims that she finally feels "normal"; that she has found her true self.

The case of Julia highlights the alteration in the sense of self that occurs under the medical intervention of psychotherapeutic drugs. Julia does not become attuned to the problems in her life, but is resituated within them. Instead of actively finding ways to change her circumstances, psychotherapeutic drugs became an easy alternative to doing so. Instead, psychotherapeutic drugs passively altered Julia's perception and correlative actions in the situations in which she found herself, making her little more than a dependent variable of the social equation which produced her mental state and her circumstances in the first place.

Julia's "success story", however, turns into a dilemma for Kramer: When Julia is taken off Prozac, she makes a claim Kramer has heard from a number of patients coming off Prozac: “I don't feel like myself anymore." This is problematic for Kramer because the change in Julia's situation came without any increased self-knowledge on her part and forces him to rethink what constitutes a "self". Julia's success at home and work on Prozac was thought to be a result of the neurochemical system within her brain. On the surface, Julia was the same woman as before going on Prozac, only with an alteration in her neurochemical systems. Of all the possible psychotherapeutic drugs, Prozac explodes 
in popularity in the early nineties for one reason: Its ability to change personality in socially appealing ways. Patients on Prozac (as with the SSRIs made in its image shortly afterward) were more active, energetic, sociable, easy going and able to deal with "everyday life". However, if SSRIs so easily change the selfhood of individuals, what does this imply about the permanence of the self in the first place?

Kramer, being a biological psychiatrist, cannot reconcile individual sense of self with the society of which it is a part; thus, he comes to endorse "cosmetic pharmacology", greatly trivializing and underestimating the implications of it. Kramer cannot see the Fascistic and Totalitarian impulses in such a practice. Instead, he goes the way of biological psychiatry discussed in chapter one and asks how the medical community, doctors and psychiatrists alike, "diagnose" symptoms of withdrawal from psychotherapeutic drugs without extending the "penumbra" of existing medical conditions further into the domain of the human condition itself. Indeed, Kramer has hit upon, as a psychiatrist, what Hacking, a philosopher of science, had called "classificatory looping", or more generally "dynamic nominalism" which was discussed in chapter one. Without recognizing it as such, the DSM includes normal reactions to everyday life, all of which are not resolved by drug use but augmented by it. The creation of psychotherapeutic drugs not only alters individual actors in response to the social situations they find themselves in, but also alters medical perception of patients and how to diagnose certain complications in health.

\section{Technology}

On psychotherapeutic drugs, selfhood is reconstituted by the medical community by favoring one existential analytic over another: Mood over Understanding. 
Psychotherapeutic drugs have the effect of making patients and their ability to negotiate their way individually in the world through autonomy over their health (altering it through struggle, coping and transcendence), things in need of medical intervention. In this way, Being itself is "enframed" through the use of medical technology. Enframing is a mode of understanding that in its revealing challenges human understanding. For Heidegger, technology constitutes a new type of cultural system that restructures the entire social world as an object of control. This instrumentalization of human beings and society comes to shape the whole of social life. Through ontic conceptualizations of the body and its illness, and now through the creation of vast numbers of psychotherapeutic drugs, human Beings themselves have fallen victim to the imperialism of technology. Human beings become viewed as aspects of technology itself: Being becomes a form of technology.

For Heidegger, technology is a way of revealing, a form of truth. Dasein (human existence including the unrealized possibilities inherent to it) is characterized as a being that can reflect on Being itself; I have been trying to show how this reflection is altered and reified by sources outside itself. The modern effect of technology on Dasein is that the world itself has become a picture to be manipulated and reformed according to the needs of the essence of technology itself: ordering for its own sake. The goal of technology, as a logical outcome of the dominance of science and an ontic worldview, is the more and more flexible and efficient ordering of resources, not as objects to satisfy our desires, but simply for the sake of ordering itself. As Heidegger writes in his famous essay "The Question Concerning Technology": "Everywhere everything is ordered to stand by, to be immediately at hand, indeed to stand there just so that it may be on call for 
a further ordering. Whatever is ordered in this way... we call standing-reserve...

Whatever stands by in the sense of standing-reserve no longer stands over against us as object" (Heidegger 1977: 17). The standing reserve, however, is no longer simply natural resources, but humanity itself.

For Heidegger, technology is a "challenging forth" of truth which forces humanity in its being-in-the-world to question its relationship to technology. We cannot affirm or deny technology without succumbing to and admitting its presence in our life. Because the essence of modern technology, according to Heidegger, is simply to grip things so as to have power over them, human beings are in danger of losing any possibility of retrieving an openness to Being that could expose other, possibly richer and fuller ways of being-in-the-world. Heidegger's main concern is that with complete mastery over the world (and now through the proliferation of psychotherapeutic drugs, other human beings and our own sense of self) we will become so entrapped in technology that we are blind to the manifold possibilities of how to experience ourselves and the world in which we live (it is here that Foucault's emphasis on discursive practices originates in importance for him. For Heidegger, an awareness of life led on the periphery that can critique and understand "totalized" forms of existence, was the only hope of overcoming such a thing as technological domination).

Foucault's technologies of the self are very dependent on Heidegger's view of technology. The theory of technologies of the self states that through certain confessional practices, subjects are made to reveal truths about themselves so they can be called forth and understood by sources of power moving through and over them. For Foucault, technologies of power reflexively modify and condition the conduct of individuals, of 
selves. Technologies of the self, however, "permit individuals to effect by their own means, or with the help of others, a certain number of operations on their own bodies and souls, thoughts, conduct and way of being, so as to transform themselves in order to attain a certain state of happiness, purity, wisdom, perfection or immortality" (Foucault 1997: 223). The intersection of the two matrices of knowledge composed of technologies of domination of others, and technologies of domination of the self is what Foucault calls “governmentality".

This sort of revelatory practice is found most strikingly on pharmaceutical manufacturer Web sites. Individual Web sites for the leading SSRI's Prozac, Paxil and Zoloft all come with self-assessment tests where individuals can score themselves for depression, social anxiety disorder and the various other types of mental disorders the particular SSRI may treat. These tests are claimed to be for "information purposes only". Visitors must agree to a waiver of liability notice before taking these tests, claiming that the manufacturer is not liable for any emotional damages or personal injuries that may result from taking the test. The Web sites go on to conclude that diagnosis and treatment of depression and other psychiatric disorders can be performed only by a doctor or qualified mental health professional. Prozac's waiver of liability reads: "The screening tool on this website is intended only for the purpose of identifying some symptoms of depression disorders, and is not designed to provide a diagnosis or treatment. Neither the screening tool, nor any other information on this Web site, is a substitute for diagnosis or treatment for depression or other psychiatric illnesses by a doctor or qualified mental health professional." However, this does not prevent Eli Lilly, the makers of Prozac, from offering the service so patients can go to their doctor or 
psychiatrist with the idea that they may be suffering from a mental disorder before they are even "officially" diagnosed. These self-assessment tests are like "pre-confession confessions" to oneself before one arrives at a doctor's office.

The Eli Lily self-assessment test for depression is a series of 20 questions trying to gauge the individual's emotional connection to the interpersonal events in their life as well as their own conceptualizations of their place within them. 18 of the 20 questions begin with the personal pronoun "I", implying that the impetus for depression lies within the individual and should not be thought of as a social condition. Further, all questions are very general, for example: "I feel downhearted, blue or sad...", "I find it easy to do the things I used to do...". The possible answers to the questions are only four: Not Often; Sometimes; Often; All The Time. Answering all of the questions as neutrally as possible, choosing "sometimes" for all twenty questions, one comes out with a score of 50 - the lower bound score for depression and the recommendation to print the result and consult a physician.

The Paxil self test for generalized anxiety disorder is even less comprehensive. The GAD test is only three questions long. The first question asks: "Do you worry excessively or are you anxious most of the time?" Nowhere does the Web site clarify or define what "worrying excessively" may constitute or what the definition of "anxiousness" is, presumably to include as many understandings of these things as possible, though as has been shown, even the DSM would not have provided a very large subtraction from this. The second question attempts to gauge physical symptoms that could be the result of GAD - restlessness, tenseness, sleeplessness etc. However, as with the Prozac test, if all questions are answered as "no" except for the last question asking 
how much anxiety interferes with one's work and life (answering it as sometimes), the end diagnosis is inconclusive and the recommendation of seeing a physician is offered.

The Zoloft self quiz is more concerned with duration of symptoms. Like the previous tests it is almost impossible to take and not be recommended to see your doctor. Of the nine questions, only one need be answered as "several days", out of three other options "not at all", "more than half the days" and "nearly every day" for the test to conclude that it is possible that one is suffering from the mental disorder concerned. One of the questions, "poor appetite or overeating" could be the result of innumerable causal factors, yet is still deemed a measure of possible diagnosis of mental disorder.

What all these self-assessment tests have in common is the creation of a subjectivity which thinks of itself as being unhealthy or on the verge of sickness. Individuals freely come to see themselves as being mentally ill through their own means. However, these tests, along with the very disorders they claim to be testing for, are questionable at best. These tests are merely one aspect of the artificial perpetuation of mental disorder throughout society by both the medical community and the pharmaceutical industry. Through the use of modern technologies, human beings are themselves an aspect of the truth it reveals - an aspect that does not include their own individual understandings of what their own lives could possibly mean on an individual level. The technologies of the self characteristic of talk therapy found within Freudian psychiatry have become obsolete to the techne of human biology. While it opens up new opportunities for human action, it correlatively decreases opportunity for human understanding. If, for Heidegger, our individual moods reveal our situatedness in the world and provide the optics through which things "show up" and matter to us in life, 
removal or chemical alteration of Mood through psychotherapeutic drug use, allows for the increasing need for technologies outside us to intervene upon us and dictate the understanding needed for an interpretation of the things that mood reveals in our lives. Indeed, individuals are giving up their freedom because the very conditions for our freedom are being removed through psychotherapeutic drugs - our ability to understand and orient ourselves within the world, of which mood is the key determinant and initiator. Throughout, I have used the word "our" to denote the presence of psychotherapeutic drug use, implying that somehow everyone is on these drugs. However, psychotherapeutic drug use is a small, though increasingly more significant, aspect of a larger, more encompassing movement of technological and medical power throughout modern society which seeks precisely what psychotherapeutic drugs achieve the alteration of the ground of what it means to be human through medical intervention. In a society dominated by science and medicine, human socialization becomes itself a thing to be altered and manipulated by these phenomena themselves. The very essence of what it means to be human is being reconstituted. In modern society, life itself has become an obstacle to living.

For Heidegger, the unifying analytic of what it means to be is Care. The world in which we live, whether we affirm or deny it, matters to us in some way. We cannot escape being affected by the things in the world and our place within it. The answer to Heidegger's seemingly grand and imposing question: what does it mean to Be at all? is not imposing at all. What it means to be is simply to Care. Our own existence is an issue for us. We are assigned the task of being someone, and the way in which we choose to deal with the possibilities that present themselves to us come irrevocably to determine 
who we are as individuals. As Heidegger writes: "Dasein is always only that which it has chosen itself to be." Through such emotions as anxiety, dread and sadness etc. human Beings become aware of their existence and their ability to take a stand on it. When these aspects of the very ground of what it means to become human are altered through psychotherapeutic drugs, humans inevitably lose the single most important determinant of what it means to Be human in the first place.

In the "Minimal Self" Lasch writes: "With the help of an elaborate network of therapeutic professions, which themselves have largely abandoned approaches stressing introspective insight in favour of "coping" and behaviour modification, men and women today are trying to piece together a technology of the self, the only apparent alternative to personal collapse." (Lasch, 1984, pg. 58). As a last resort in a world that offers little comfort against the demands of work and life, individuals are resorting to the very thing that makes their lives without comfort or meaning in the first place - the nihilism of living by values over and above individual human control as represented by medicine, technology and capital - thereby increasing the very cause of the symptoms they are trying to alleviate. 


\section{Conclusion}

The story I have tried to tell contained two plots - nihilism and technologies of the self - which were expressing themselves through individuals and the perpetuation of ill mental health throughout society. It has been my thesis that since the end of the second World War, bio-power has intervened in the lives of individuals through the creation of psychotherapeutic technologies of the self in the form of magic bullet pills that resocialize human individuals as more active, more personable, more out-going and optimistic in what could be conceived as inherently pessimistic times. Further, I hope to have shown that mood and personality are being significantly altered through the medical technology of self-transformation induced by psychotherapeutic drugs in an attempt to reconcile emotional life and the social reality individuals are faced with on a day-to-day basis.

I have woven my way through the "creation" of mental illness as is evidenced by the APA's DSM and its revision process. At the same time, I have tried to make a case for the occurrence of "real" mental illness through the modern domination of technological nihilism - of which psychotherapeutic technologies of the self are a very part. I also spent some time analyzing the role of the pharmaceutical industry in the creation of illness and the proliferation of various copy-cat medications manufactured to "treat" illness. All these threads have contributed to the rise and mass dependence of psychotherapeutic drugs. This is our brave new world.

The horizon for this technological nihilism is vast. Currently, scientists for large biotechnology firms are trying to find "genes that fit": researchers are trying to code patients' individual genetic information, allowing them to develop more effective drugs 
and more tailored treatments for both mental and physical disorders. The next step in the brave new world of artificial mood and personality creation and manipulation is patient genetic profiling. The buzz words in the coming decade will be "personalized medicine". With readily available genetic data, scientists will be able to identify genetic difference between diseased and healthy states, offering a "perfect pill" for each individual and their ailment. Not only will this lessen the chances of counter-indications within patients, lessen the chances of what is referred to as a drug's "side-effect profile", it will also recast human personality and emotion to the dictates of social necessity. Conceivably, one day there will be no longer such a thing as pain and suffering. And without such uniquely human attributes as pain and suffering, humans will lose significant tools in the pursuit of self-understanding. Indeed, with advances in genetic engineering, perhaps we are moving beyond what could be recognized as human Being at all.

Only when individuals can reclaim autonomous control over their health and well-being, over their unique ability to suffer and interpret the meaning of their individual suffering, will the domination of bio-power no longer hold sway. Only when we understand Being as a process of overcoming, can we remove ourselves from medical technology and the nihilism it implies. As the theologian Paul Tillich once wrote, we must find the courage to $\mathrm{Be}$, the courage to find meaning and relatedness and the ability to actualize self-understanding in times that increasingly appear to mitigate against it (Tillich 1952). 


\section{References}

Diagnostic and Statistical Manual of Mental Disorders, 3rd ed. Revised. 1987. Edited by Robert et al Spitzer. Washington: The American Psychiatrc Association.

Diagnostic and Statistical Manual of Mental Disorders 4th ed. 1994. Washington: The American Psychiatric Association.

Reassessing Foucault. 1994. Edited by Colin Jones and Roy Porter. London: Routledge.

The Essential Frankfurt School Reader. 1997. Edited by Andrew Arato and Eike Gebhardt. New York: Continuum.

Foucault, Health and Medicine. 1997. Edited by Alan Peterson and Robin Bunton. London: Routledge.

A Handbook for the Study of Mental Health. 1999. Edited by Allan V. Horwitz and Teresa L. Scheid. Cambridge: Cambidge University Press.

A Report on Mental Illness in Canada. 2002. Health Canada, Ottawa, Health Canada Editorial Board Mental Illness in Canada.

Ref Type: Report

Foucault and Heidegger, Critical Encounters. 2003. Edited by Alan Milchman and Alan Rosenberg. Minneapolis: University of Minneasota Press.

Compendium of Pharmaceuticals and Specialties. 2003. Edited by Canadian Pharmacists Association. Ottawa.

IMS Health Data 2003. IMS Health Canada. 2003. Ref Type: Report

Culture and Depression. 2004. Edited by Arthur Kleinman and Byron Good. Berkeley: University of California Press.

Adorno, Theodore. 1991. The Culture Industry. London: Routledge.

Andreasen, Nancy. 1985. Broken Brain: The Revolution in Biological Psychiatry. New York: Harper Collins.

Angell, Marica. 2004. The Truth about Drug Companies. New York: Random House.

Arendt, Hannah. 1954. Between Past and Future. New York: Penguin.

Ballard, Bruce W. 1991. The Role of Mood in Heidegger's Ontology. Lanham: University Press of America. 
Bauman, Zygmunt. 2002. Society Under Siege. Cambridge: Polity.

Bocock, Robert. 1993. Consumption. New York: Routledge.

Bowker, Geoffrey C and Susan Leigh Star. 1999. Sorting Things Out: Classification and its Consequences. Cambridge: MIT Press.

Burton, Robert. 1965. The Anatomy of Depression. Edited by Lawrence Babb. Michigan: Michigan University Press.

Callinicos, Alex. 1999. Social Theory. New York: New York University Press.

Campbell, Robert M. and Leslie Pal. 1994. The Real Worlds of Canadian Politics. 3rd ed. Peterborough: Broadview Press.

Denzin, Norman. 1984. On Understanding Emotion. San Fransico: Joessy-Bass.

Desrosieres, Alan. 1990. How to Make Things Which Hold Together. In Discourses on Society. Edited by B. Wittrock R. Whitely Peter Wagner. Dordrecht: Kluwer Academic Publishers.

Diamond, Stanley. 1974. In Search of the Primitive. New Brunswick: Transaction Publishers.

Dreyfus, Hubert L. and Paul Rabinow. 1983. Michael Foucault: Beyond Structuralism and Hermeneutics. Chicago: University of Chicago Press.

Dreyfus, Hubert L. 1991. Being-in-the-World. Cambridge: The MIT Press.

Duffin, Jacalyn. 2000. History of Medicine. Toronto: University of Toronto Press.

Foucault, Michel. 1965. Madness and Civilization. New York: Vintage.

Foucault, Michel. 1970. The Order of Things. New York: Vintage.

Foucault, Michel. 1973. The Birth of the Clinic. New York: Vintage.

Foucault, Michel. 1976. Mental Illness and Psychology. Berkeley: University of California Press.

Foucault, Michel. 1980. Power/Knowledge. New York: Pantheon.

Foucault, Michel. 1994. Ethics, Subjectivity and Truth. New York: The New Press.

Foucault, Michel. 1994. Aesthetics, Method and Epistemology. New York: The New Press. 
Foucault, Michel. 1994. Power. New York: The New Press.

Freud.Sigmund. 1989. The Freud Reader. Edited by Peter Gay. New York: W.W. Norton.

Fromm, Erich. 1941. Escape From Freedom. New York: Henry Holt and Co.

Fromm, Erich. 1949. Man for Himself. London: Ark Publishers.

Fromm, Erich. 1955. The Sane Society. New York: Henry Holt and Co.

Fromm, Erich. 1976. To Have or To Be? New York: Continuum.

Gelvin, Micheal. 1989. A Commentary on Heidegger's Being and Time. revised ed. Dekalb: Northern Illinios Press.

Greenberg, Gary, "Is it Prozac? Or Placebo?," Mother Jones 28 (6): 76-86 (2003).

Hacking, Ian. 1986. Making Up People. In Reconstructing Individuality. Edited by Thomas Heller. Standford: Stanford Univerity Press.

Hacking, Ian. 1999. The Social Construction of What? Cambridge: Harvard University Press.

Hacking, Ian. 2002. Historical Ontology. Cambridge: Harvard University Press.

Harvey, David. 1990. The Condition of Postmodernity. Cambridge: Blackwell.

Healy, David. 1997. The Antidepressant Era. Cambridge: Harvard University Press.

Healy, David. 2002. The Creation of Psychopharmacology. Cambridge: Harvard University Press.

Healy, David. 2003. Let Them Eat Prozac. Toronto: James Lorimer \& Co.

Heidegger, Martin. 1962. Being and Time. San Francisco: Harper Collins.

Heidegger, Martin. 1977. The Question Concerning Technology and Other Essays. New York: Harper and Row.

Heidegger, Martin. 1993. Basic Writings. San Francisco: Haper Collins.

Held, David. 1980. Introduction to Critical Theory. Berkeley: Univeristy of California Press. 
Hersch, Edwin L. 2003. From Philosophy to Psychotherapy. Toronto: University of Toronto Press.

Horkheimer, Max. 1974. Critique of Instrumental Reason. New York: Continuum.

Horkheimer, Max and Theodore Adorno. 1994. Dialectic of Enlightenment. New York: Continuum.

Horkheimer, Max. 1999. Critical Theory. New York: Continuum.

Horwitz, Allan V. 2002. Creating Mental Illness. Chicago: University of Chicago Press.

Huxley, Aldous. 1965. Brave New World and Brave New World Revisited. New York: Harper and Row.

Illich, Ivan. 1976. Limits to Medicine. London: Marion Boyers Publishers.

Keen, Ernest. 2002. Depression. Westport: Praeger Publishers.

Kirk, Stuart A. and Herb Kutchins. 1992. The Selling of DSM. New York: Aldine De Gruyter.

Kirsch, Irving and Thomas J. Moore, "The Emperor's New Drugs: An Anaysis of Antidepressant Medication Data Submitted to the US Food and Drug Administration," Prevention and Treatment 5 (23) (2002).

Klein, Naomi. 2000. No Logo. Toronto: Vintage Canada.

Kramer, Peter. 1997. Listening to Prozac. New York: Penguin.

Kuhn, Thomas. 1996. The Structure of Scientific Revolutions. Chicago: University of Chicago Press.

Lasch, Christopher. 1979. The Culture of Narcissism. New York: W.W. Norton and Co.

Lasch, Christopher. 1984. The Minimal Self. New York: W.W. Norton.

Lee, Dorothy. 1987. Freedom and Culture. Prospect Heights: Waveland Press.

Leys, Collin. 2001. Market-Driven Politics. London: New Left Books.

Marcuse, Herbert. 1964. One-Dimensional Man. Boston: Beacon Press.

Marx, Karl and Friedrich Engels. 1978. The Marx-Engels Reader. Edited by Robert Tucker. New York: W.W. Norton and Co. 
Marx, Karl. 1988. Economic and Philosophic Manuscripts of 1844. Amherst: Prometheus Books.

Mitnick, Barry M. 1980. The Political Economy of Regulation. New York: Columbia University Press.

Moynihan, Ray and Iona Heath and David Henry, British Medical Journal (324): 886-891 (2002).

Nietzsche, Friedrich. 1967. The Will to Power. New York: Vintage.

Pattison, George. 2000. The Later Heidegger. London: Routledge.

Putnam, Robert D. 2000. Bowling Alone. New York: Simon and Schuster.

Rifkin, Jeremy. 2000. The Age of Access. New York: Putnam.

Rosenhan, David, "On Being Sane in Insane Places," Science (179): 250-258 (1973).

Roszak, Theodore. 1972. Where the Wasteland Ends. New York: Double Day and Co.

Shorter, Edward. 1997. A History of Psychiatry. New York: John Wiley and Sons.

Spitzer, Robert L., "On Pseudoscience in Science, Logic in Remission and Psyhciatric Diagnosis: A critique of Rosenhan's "On Being Sane in Insane Places."," Journal of Abnormal Psychology 84: 442-452 (1975).

Spitzer, Robert L. 1980. Introduction to Diagnostic and Statistical Manual of Mental Disorders. Washington: American Psychiatric Association.

Spitzer, Robert L. et al, "Research Diagnostic Criteria," Archives of General Psychiatry 35: 773-782 (1978).

Spitzer, Robert L. et al. 1983. Classification in Psychiatry. In Comprehensive Textbook of Psychiatry. Edited by Harold I. Kaplan and Benjamin Sadok. Baltimore: Williams \& Wilkins.

Thompson, John and Patricia Barid and Jocelyn Downie. Report of the Committee of Inquiry on the Case Involving Dr. Nancy Olivieri, the Hospital for Sick Children, the Univeristy of Toronto, and Apotex Inc. 2001.

Ref Type: Report

Tillich, Paul. 1952. The Courage To Be. London: The Fontana Library.

Wakefield, Jerome C., "The Concept of Mental Disorder," American Psychologist 47 (3): 373-388 (1992). 
Wakefield, Jerome C., "Disorder as Harmful Dysfunction: A Conceptual Critique of DSM-III-R's Definition of Mental Disorder," Psychological Review 99 (2): 232-247 (1992). 